ANL-5934

Health and Safety TID -4500 (14th ed.)

AEC Research and

Development Report

ARGONNE NATIONAL LABORATORY

P.O. Box 299

Lemont, Illinois

ENVIRONMENTAL RADIOACTIVITY AT

ARGONNE NATIONAL LABORATORY

Report for the Year 1957

Written by

J. Sedlet

Experimental Work Done by:
C.D. Eldred
F.S. Iwami
M. T. Epifani
J. Sedlet
K. K. Gallagher
E. Wood
C. M.Green, Jr。

Industrial Hygiene and Safety Division

Submitted for Publication in:

October, 1958

\footnotetext{
Operated by The University of Chicago under

Contract $W-31-109-$ eng-38
} 


\section{DISCLAIMER}

This report was prepared as an account of work sponsored by an agency of the United States Government. Neither the United States Government nor any agency Thereof, nor any of their employees, makes any warranty, express or implied, or assumes any legal liability or responsibility for the accuracy, completeness, or usefulness of any information, apparatus, product, or process disclosed, or represents that its use would not infringe privately owned rights. Reference herein to any specific commercial product, process, or service by trade name, trademark, manufacturer, or otherwise does not necessarily constitute or imply its endorsement, recommendation, or favoring by the United States Government or any agency thereof. The views and opinions of authors expressed herein do not necessarily state or reflect those of the United States Government or any agency thereof. 


\section{DISCLAIMER}

Portions of this document may be illegible in electronic image products. Images are produced from the best available original document. 
TABLE OF CONTENTS

$\underline{\text { Page }}$

I. SUMMARY ..................... 3

II. PROGRAM AND PROCEDURES. . . . . . . . . . . . 9

III. RADIOACTIVITY IN ENVIRONMENTAL SAMPLES. . . . . 13

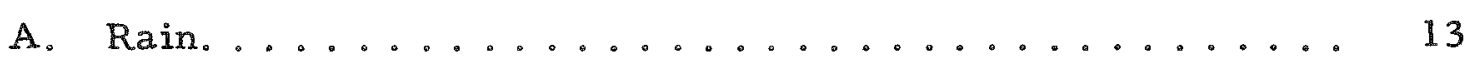

B. Air Filters ....................... 14

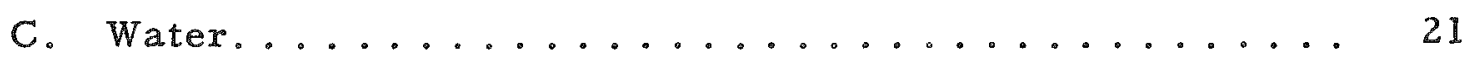

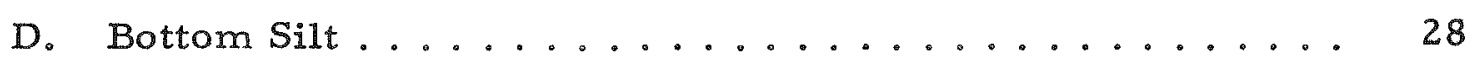

E. Surface Soil. ...................... 35

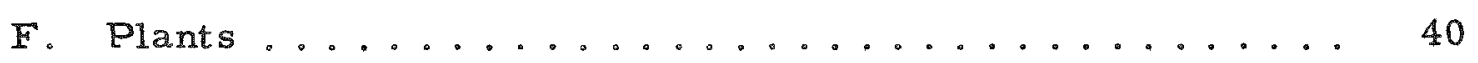

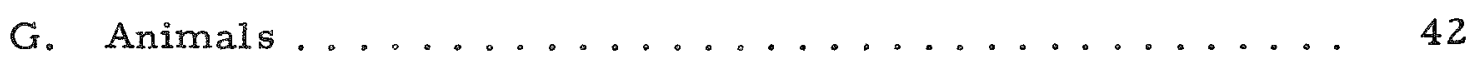




\title{
ENVIRONMENTAL RADIOACTIVITY AT ARGONNE NATIONAL LABORATORY
}

\author{
Report for the Year 1957
}

\section{SUMMARY}

Concentrations of radioactivity in several types of materials collected from the ANL site and from locations approximately 10,25, and 100 miles from the Laboratory are given in this report. The principal purpose of this work was to determine if any radioactive materials were released to the environment by Argonne National Laboratory. In order to evaluate this, it was also necessary to determine the natural concentration of radioactivity in the environment and the contribution of fission product fallout to the total activity.

The sampling locations on and near the site are given in Part II. Since ANL waste water is discharged into Sawmill Creek, which in turn empties into the Des Plaines River, special emphasis was placed on sampling these streams. Most of the results were obtained by counting the total alpha and beta activities after suitable preparation of the samples: some of the samples were also analyzed for specific radioactive elements and nuclides. The average total alpha and beta activities found in water, lake and stream beds ("bottom silt"), soil, and plants during 1957 are summarized in Figures 1-3. For purposes of comparison, Figures $4-7$ give the results obtained on similar samples from 1952 through 1957. The long-lived alpha and beta activities found in air filter samples from 1952-57 are given in Section III-B of this report.

Fission product activity from fallout was found in all types of samples from most locations, with the maximum amount of fallout occurring from July through October. Radioactivity originating at ANL was found in water and bottom silt obtained from Sawmill Creek below the Argonne waste-water outfall. Water from this location contained an average of 12 micromicrocuries $(\mu / \mathrm{c})$ of alpha activity per liter, or 9 k $\mu \mathrm{c} / 1$ higher than water collected from Sawmill Creek above the Laboratory site. This increase is attributed primarily to uranium added to the stream in ANL waste water. About $90 \%$ of the samples obtained below outfall contained amounts of uranium above normal. The average uranium concentration in water from this location was $23 \mu \mu \mathrm{c} / 1$, or $0.3 \%$ of the maximum permissible concentration (MPC)*

\footnotetext{
* The concentrations of radioactivity attributable to Aruonke operations were quite low, but it may be of interest to compare them with some published maximum permissible concentrations. The MPC's used in this report are those given in Title 10, Part 20, of the Federal Register and apply to the dissemination of radioactivity to unrestricted areas by installations licensed by the AEC. For most nuclides the values given in the Federal Register are 10\% of those given in National Buredu of Standards Handbook. 52, and, therefore, usually agree with recommendations made by the International Committee on Radjological Protection. The MPC's for water apply to drinking water only, and it shonld be emplasized that sawmill Creek water is not used for human consumption.
} 
Figure 1

AVERAGE RADIOACTIVITY IN SURFACE WATER, 1957

Sawmill Creek, Above Site

Sawmill Creek, Below Site

ANL Ponds

DesPlaines River, Above Site

Desplaines River, Below Site

10 and 25 Mile Zones

100 Mile Zone

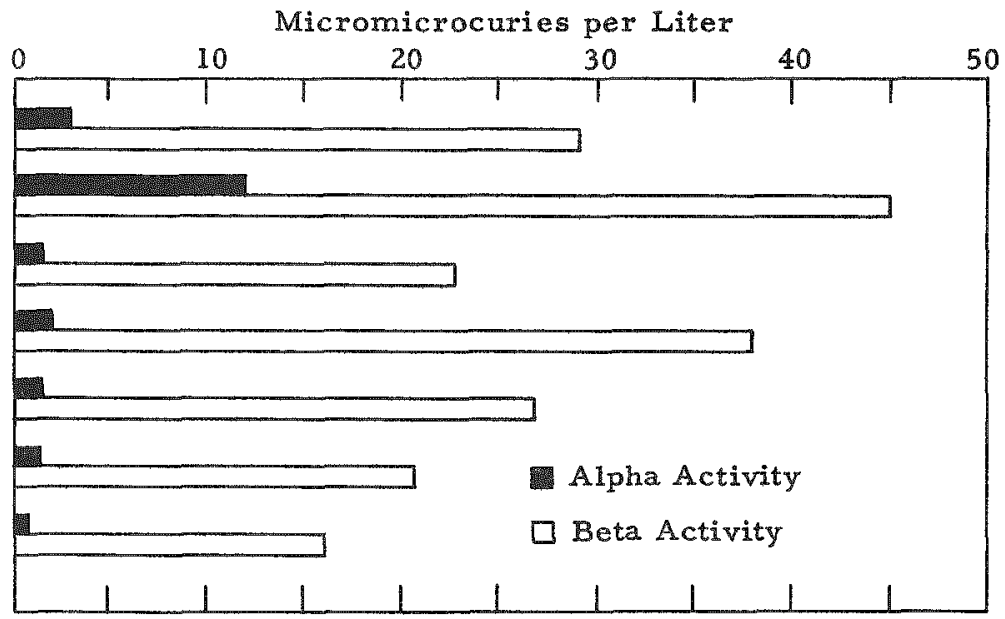

Figure 2

AVERAGE RADIOACTIVITY IN BOTTOM SILT, 1957

Sawmill Creek, Above Site

Sawmill Creek, Below Site

ANL Ponds

DesPlaines River, Above Site

DesPlaines River, Below Site

10 and 25 Mile Zones

100 Mile Zone

Micromicrocuries per Gram

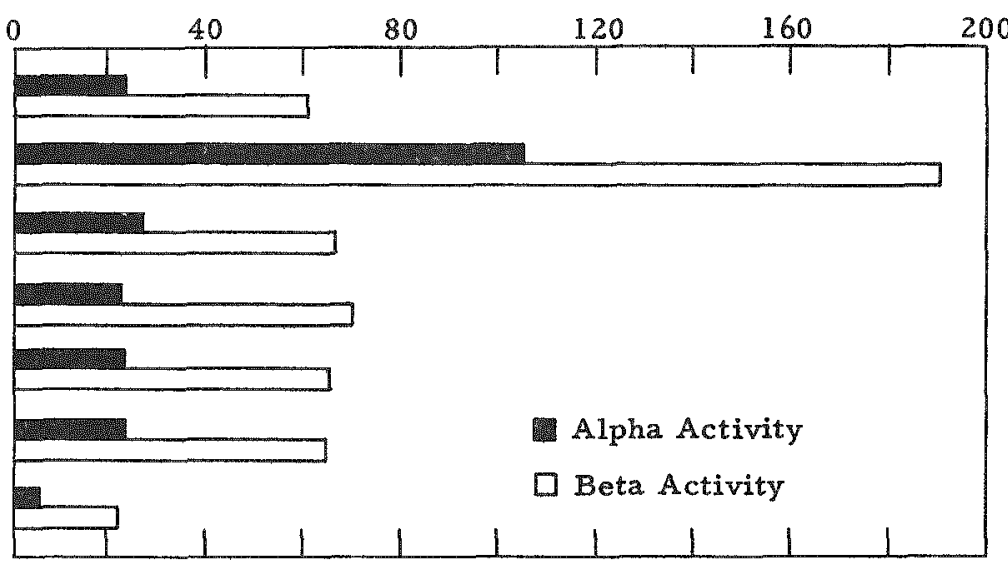

Figure 3

AVERAGE RADIOACTIVITY IN SOIL AND PLANTS, 1957

Micromicrocuries per Gram

Soil

ANL Site

10 and 25 Mile Zones

100 Mile Zone

Plants

ANL Site

10 and 25 Mile Zones

100 Mile Zone

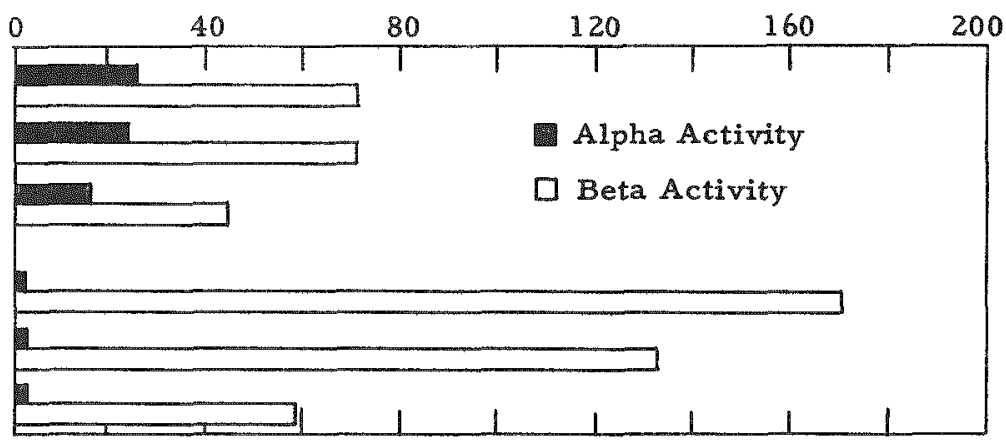


Figure 4

AVERAGE RADIOACTIVITY IN SURFACE WATER, 1952-57

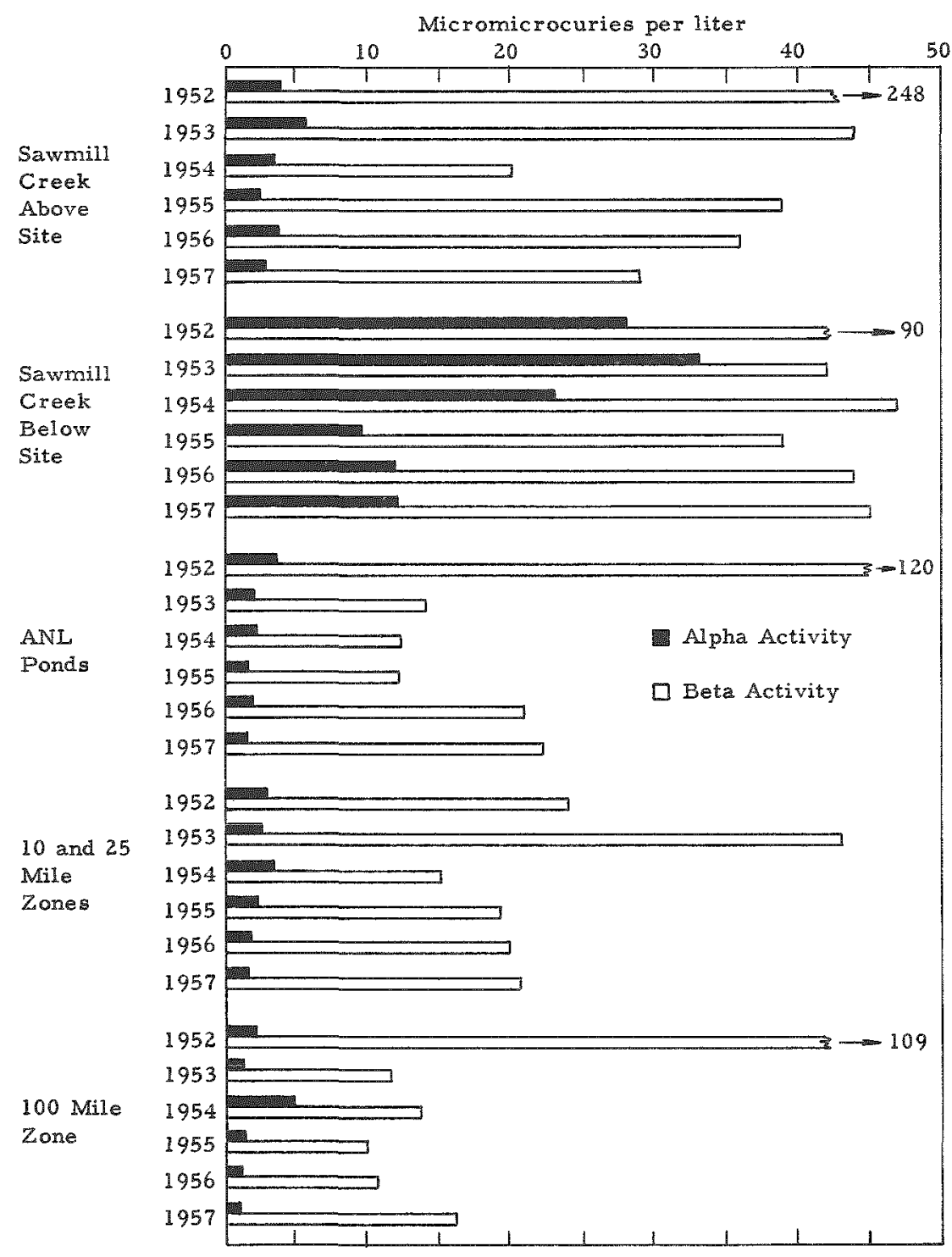

Figure 5

AVERAGE RADIOACTIVITY IN BOTTOM SILT, 1952-57

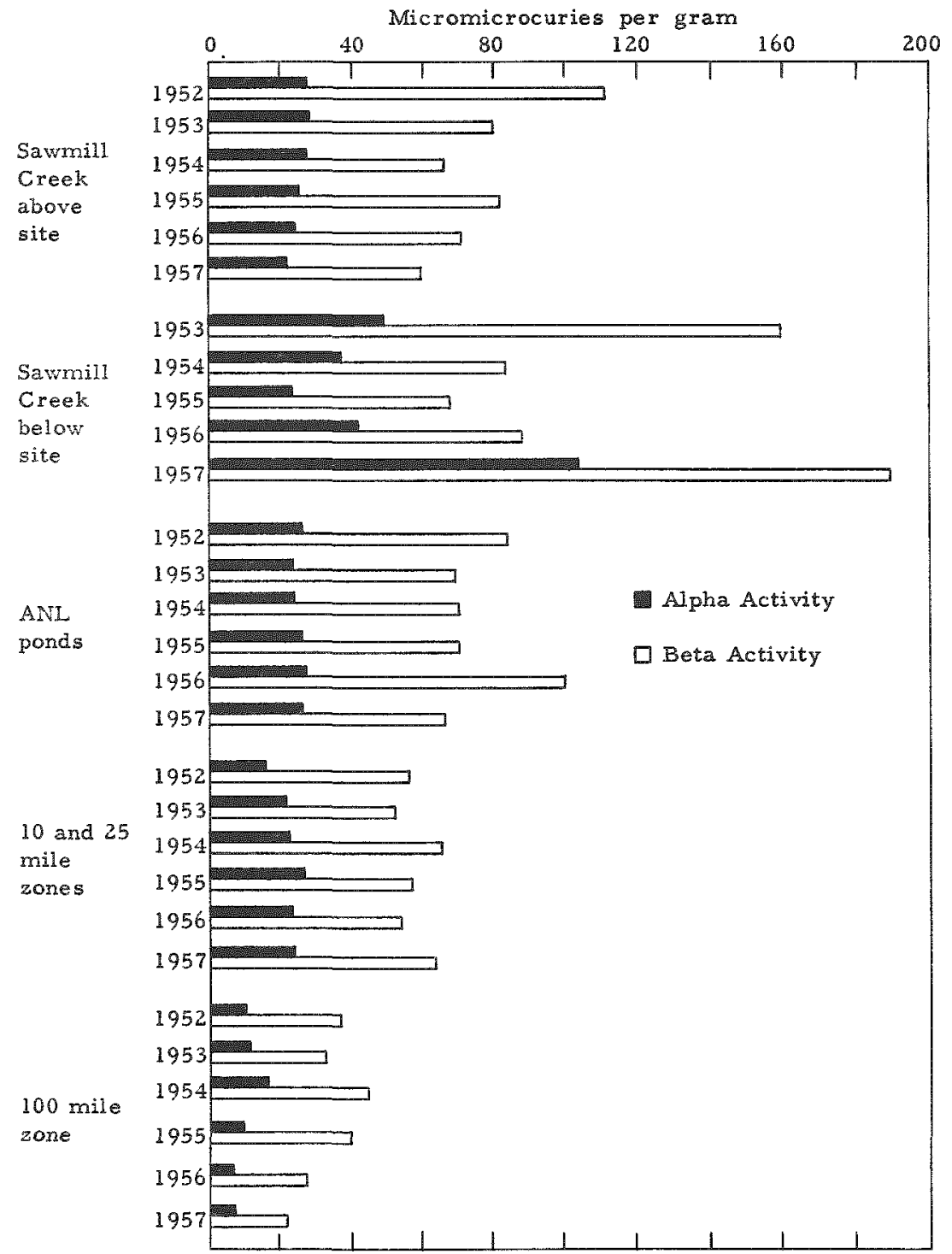


Figure 6

AVERAGE RADIOACTIVITY IN SURFACE SOIL, 1952-57

Micromicrocuries per Gram

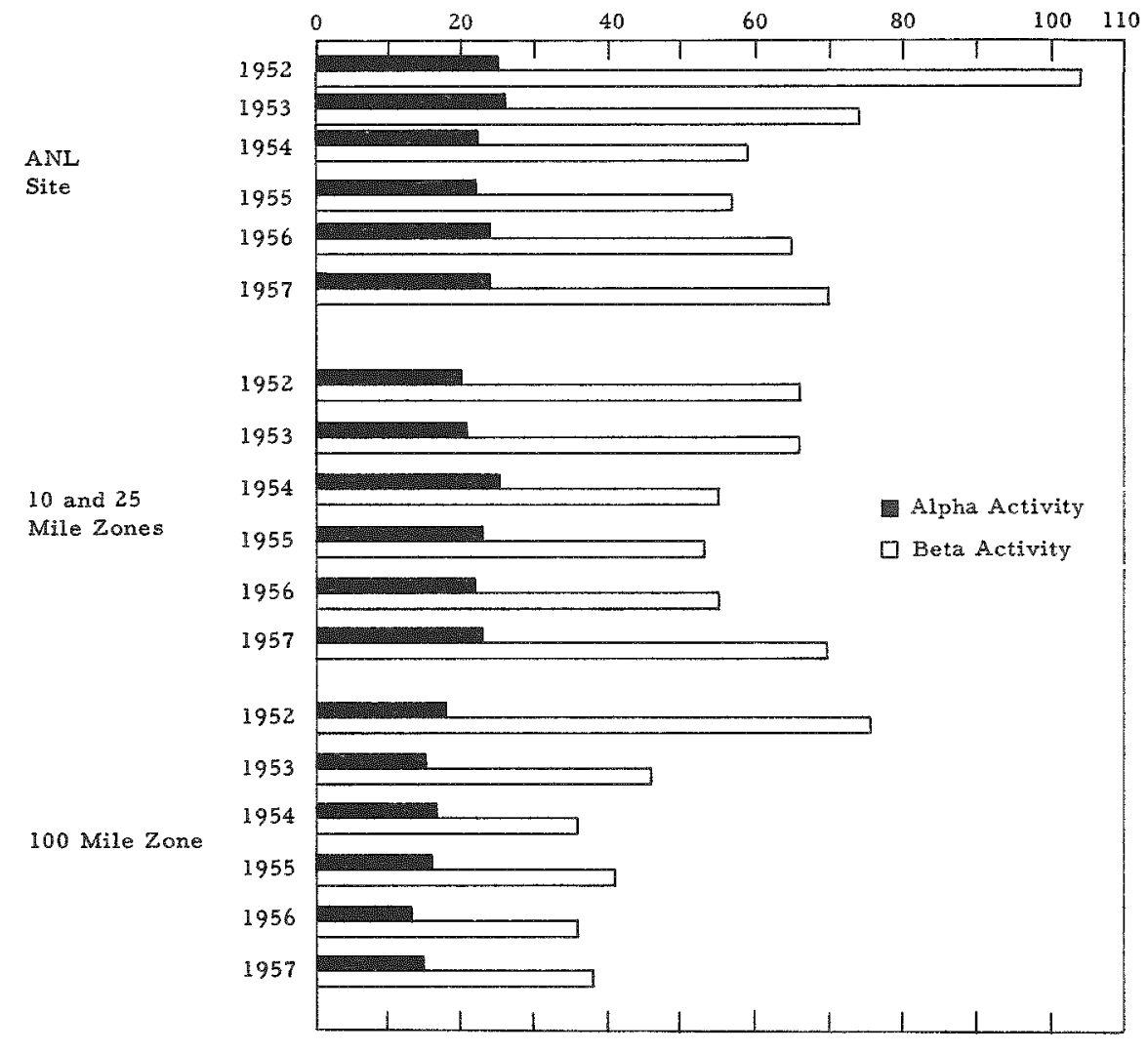

Figure 7

AVERAGE RADIOACTIVITY IN PLANTS, 1952-1957

Micromicracuries per gram

ANL

Site

ANL
Site

10 and 25

Mile Zones

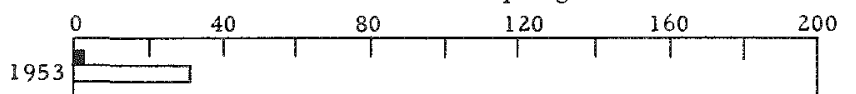

$1954 \longrightarrow$

1955

$1956 \longrightarrow$

$1957+$

1953

$195+\longdiv { 2 }$

$\square$ Beta Activity

1955

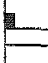

100 Mile

$1 9 5 6 \longdiv { \square }$

$1957 \longrightarrow$

$1 9 5 3 \longdiv { - }$

$1954 \longrightarrow$

Zone

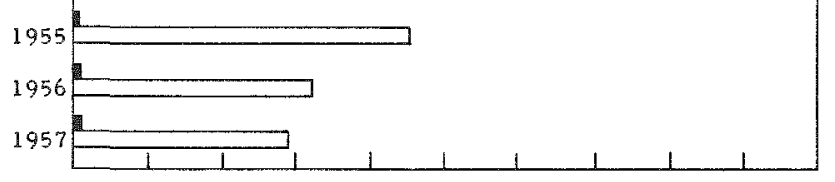


for drinking water. A smaller contribution to the alpha activity in belowsite water was made by plutonium added to the Creek in ANL waste water. The average plutonium concentration at this location was $2.3 \mu \mu \mathrm{c} / 1$. This average value was $1.4 \%$ of the MPC, while the maximum plutonium concentration found, $25 \mu \mu \mathrm{c} / 1$, amounted to $16 \%$ of the MPC. Plutonium was not detected in any of the above-site samples.

Fission products from fallout were found in Creek water from both locations; beta activity from Laboratory waste water was found only below the outfall. The total beta activity averaged $45 \mu \mu \mathrm{c} / 1$ in below-site water and $29 \mu \mu \mathrm{c} / 1$ in above-site water. The average contribution of fallout and ANL waste water to the total beta activity in the stream was approximately $15 \mu \mu \mathrm{c} / 1$ from each source. The remaining activity is attributed to naturally occur ring radioactive nuclides, including $\mathbb{K}^{40}$.

Concentrations of $5 r^{90}$ greater than $2 \mu \mu \mathrm{c} / 1$ were found only in below-site water This nuclide was added to the Creek in ANL waste water. The arrerage $\mathrm{Sr}^{90}$ concentration, $3.0 \mu \mu \mathrm{c} / 1$, was about $4 \%$ of the MPC. Other fission products were found at both locations and were contributed at various times by both fallout and Laboratory waste water. The individual results and the number of samples in which each nuclide was derected are given in Part III.

The activity added to Sawmill Creek in ANL waste water and fallout had no detectable effect on the radioactivity in the Des Plaines River water. The average total alpha activities (about $1.9 \mu \mu \mathrm{c} / 1$ ), uranium concentrations (1.4 $\mu \mu \mathrm{c} / 1$ ), and beta activities (about $33 \mu \mu \mathrm{c} / 1$ ) were essentially the same above and below the ANL site.

The activities found in the natural ponds on the ANL site were not significantly different from those in lakes and streams near the Laboratory. Concentrations of total alpha activity averaged $1.4 \mu \mu \mathrm{c} / 1$ in the on site and $1.5 \mu \mu \mathrm{c} / 1$ in the off-site samples. The corresponding results for beta activity were 22 and $21 \mu \mu \mathrm{c} / 1$. Similar results were obtained in previous years. The storage lagoon for contaminated waste water on the ANL site contained $2-3 \mu \mu \mathrm{c} \alpha / 1$ and $10-50 \mu \mu \mathrm{c} \beta / 1$ in excess of normal surface water. However, many water samples containing similar concen trations of beta activity derived from fallout were also found during the year. The total activities in water collected from the reference sites ranged from 0.1 to $1.5 \mu \mu \mathrm{c} \alpha / 1$ and 3.7 to $80.3 \mu \mu \mathrm{c} \beta / 1$. Samples containing the higher concentrations of beta activity decayed at a more rapid $r$ ate than the lower samples, indicating a larger amount of fission product activity in the former. No important changes from earlier years were noted.

The total activities found in bottom silt varied considerably with location and time of sampling. Samples containing sand and other relatively large particles were generally lower in activity than material 
consisting primarily of clay. Some of the samples collected from Sawmill Creek below the waste-water outfall contained activity added to the Creek in ANL waste water. These samples contained from 61 to $398 \mu \mu \mathrm{c} \alpha / \mathrm{g}$ and from 110 to $1157 \mu \mu \mathrm{c} \beta / \mathrm{g}$, as compared with normal values of $10-30 \mu \mu \mathrm{c} \alpha / \mathrm{g}$ and 50-90 $\mu \mu \mathrm{c} \beta / \mathrm{g}$. The presence of contamination in these samples was confirmed by additional analyses on the two below site samples of highest activity. The latter contained 85 and $101 \mu \mathrm{gU} / \mathrm{g}, 93$ and $149 \mu \mu \mathrm{cPu} / \mathrm{g}$, and 8.4 and $13.5 \mu \mu \mathrm{cSr} 90 / \mathrm{g}$. Bottom silt from other locations contained about $2.5 \mu \mathrm{gU} / \mathrm{g}$ and less than 0.3 and $1.5 \mu \mu \mathrm{c} / \mathrm{g}$ of plutonium and $\mathrm{Sr}^{90}$, respectively. As was found for flowing water, the increased activity in Sawmill Creek had no significant effect on the radioactive content of the Des Plaines River bed. Bottom silt from the Des Plaines River averaged $21 \mu \mu \mathrm{c} \alpha / \mathrm{g}$ and $69 \mu \mu \mathrm{c} \beta / \mathrm{g}$ above the ANL site and $23 \mu \mu \mathrm{c} \alpha / \mathrm{g}$ and $64 \mu \mu \mathrm{c} \beta / \mathrm{g}$ below site.

The bed of the storage lagoon for contaminated waste water contained 10 to 20 times the normal concentration of alpha and beta activities as a result of the use made of this lagoon. Bottom silt from the natural lagoons on the ANL site and from the lakes and streams near the Laboratory averaged about 25 and $65 \mu \mu \mathrm{c} / \mathrm{g}$ of alpha and beta activities respectively. These values were similar to those found in previous years. The average alpha activity in the reference site samples was $6 \mu \mu \mathrm{c} / \mathrm{g}$, approximately the same as found in 1955 and 1956. The average beta activity, $21 \mu \mu \mathrm{c} / \mathrm{g}$, was about $15 \mu \mu \mathrm{c} / \mathrm{g}$ less than the average for the years $1952-$ 1956 , but the total beta activity is, of course, greatly influenced by the amount of fallout at the time of sampling.

Most soil samples contained normal activities: $20-30 \mu \mu \mathrm{c} \alpha / \mathrm{g}$ and $40-80 \mu \mu \mathrm{c} \beta / \mathrm{g}$. Increases in beta activity of 25 to $150 \mu \mu \mathrm{c} / \mathrm{g}$ due to fallout activity were found in some of the samples collected on and near the site during June, August and October. The beta activities in these samples decayed to normal values in about four months. One soil sample, collected on the Laboratory site near a uranium storage shed, contained approximately $20 \mu \mu \mathrm{c} / \mathrm{g}$ in excess of the normal uranium concentration. Except for this sample, no evidence of contamination originating at Argonne was found in soil.

The alpha activities in grass during 1957 were uniformly low, averaging $1.6 \mu \mu \mathrm{c} / \mathrm{g}$ at all locations. The beta activity in grass collected on and near the ANL site averaged 170 and $132 \mu \mu \mathrm{c} / \mathrm{g}$, respectively. This was about $70 \mu \mu \mathrm{c} / \mathrm{g}$ greater than found in other years; this increase was evidently due to above-normal concentrations of fission product fallout in samples collected in April, March, August, and October. The beta. activities in all samples decayed to normal values $(40-80 \mu \mu \mathrm{c} / \mathrm{g}) \mathrm{regard}$ less of the original activity. Grass from the reference sites contained an average of only $57 \mu \mu \mathrm{c} \beta / \mathrm{g}$. However, samples were not collected from the reference sites during the months when increased beta activity was found on and near the site. 
Airborne particulate radioactivity was collected at five locations on the Argonne site and, beginning in May, 1957, at three locations near the Laboratory. The activities remaining on the filter papers after the decay of thoron and radon daughters were due primarily to fission products from nuclear detonations (for most of the beta activity) and to longlived naturally occurring radioactive nuclides present in dust in the air (for the alpha activity and a small fraction of the beta activity). The alpha activities have remained quite constant. The average annual alpha activity three days after sample collection ranged from 0.007 to 0.010 micromicrocurie per cubic meter $\left(\mu \mu / \mathrm{M}^{3}\right)$ from 1953 to 1957. The beta activity was more variable. The average beta activities in the weekly samples were $1.2 \mu \mu \mathrm{c} / \mathrm{M}^{3}$ in 1953 and $0.4 \mu \mu \mathrm{c} / \mathrm{M}^{3}$ in 1954. Since 1954 the beta activity increased approximately $0.6 \mu \mu \mathrm{c} / \mathrm{M}^{3}$ per year to an average of $2.1 \mu \mu \mathrm{c} / \mathrm{M}^{3}$ in 1957, probably because nuclear detonations were carried out over longer periods of time in recent years. Short-term increases in beta activity, accompanied by an increase in the decay rate, were observed during all years. These increases were, therefore, due to fission products of recent origin.

No consistent or important differences between the off-site and onsite air filter samples were noted, and no significant concentrations in air due to ANL operations were found. The alpha and beta activities at all locations were about $0.2 \%$ of the MPC for unknown mixtures of activities.

\section{PROGRAM AND PROCEDURES}

This report presents the results of the environmental monitoring program at Argonne National Laboratory for the year 1957, together with some of the results obtained from air samples collected earlier. The purposes of the program are to evaluate the natural radioactive contents of the ANL site and its environment, and to determine the magnitude and origin of any radioactivity above the natural levels. Of particular interest is the detection of any radioactive materials released to the environment by Argonne National Laboratory. The radioactive content of the environment was measured by performing radiochemical analyses and total activity counting on the numbers and types of samples given in Table I.

\section{TABLE I}

\section{SAMPLES COLLECTED IN 1957}

\begin{tabular}{|ll|lr|}
\hline Water & 349 & Plant & 100 \\
Precipitation & 122 & Air Filter & 644 \\
Soil & 109 & Animal & 13 \\
Bottom Silt & 162 & & \\
\hline
\end{tabular}


Since the most probable means of dispersal of radioactive contamination are by water and air, most of the samples were of these types. Samples were collected from the Argonne site and from locations approximately 10,25 , and 100 miles from the Laboratory, as indicated in Figures 8 and 9. Samples collected from the 10 and 25-mile locations ("near" the Laboratory) were expected to indicate the extent and direction of radioactive contamination in the event that significant amounts of activity had been released as a result of operations at Argonne. Samples from the $100-$ mile locations (termed reference sites) were originally intended to serve as cono tinuous checks for sample contamination during collection, analysis, and storage. These purposes have been realized for alpha-activity measurements, but, as a result of generalized fission product fallout, beta-activity measurements at the levels encountered were more difficult to interpret. The latter measurements have served to indicate the extent and magnitude of fission product fallout; but by making the proper comparisons between off-site and on-site samples, by measuring beta-activity decay rates, and by analyzing for fission products of various half-lives, it was usually possible to distinguish between beta activity from nuclear explosions, Argonne operations, and natural sources.

The measurements of total activity were obtained by counting thick samples (10 to $75 \mathrm{mg} / \mathrm{cm}^{2}$ ) in nylon-windowed alpha and beta counters. For all samples except air filters, counting rates were converted to disintegration rates by applying corrections determined for $\mathrm{Pu}^{239}$ (for alpha particles) and $\mathrm{Tl}^{204}$ (for beta particles) under the same conditions used in counting samples. The results obtained in this way represent the true disintegration rates only if the particles emitted by the samples have the same energies as those emitted by $\mathrm{Pu}^{239}$ and $\mathrm{Tl}^{204}$. True disintegration rates cannot be obtained from measurements of total activity, since the samples contained mixtures of nuclides of varying and unknown composition and at low concen trations. However, these measurements of total activity can be made rapidly and are useful in the intercomparisons of samples and in determining which samples should be analyzed for specific elements and nuclides.

The long-lived activities given for air filters in Section III-B were obtained by applying correction factors measured for radon daughters to the counting rates. The disintegration rates obtained in this way represent the radon concentrations in air that would give rise to the observed counting rates on the filters papers. As was the case for the other types of samples, true disintegration rates cannot be obtained for air filter samples by total alpha and beta counting. How ever, this type of counting permits the rapid analysis of large numbers of samples and the results are useful in the intercomparison of samples and in the comparison of long-lived activity with radon concentration in the air.

Specific fission product analyses were made by separating the desired element with carrier added and counting in an anti-coincidence 


\section{Figure 8}

Sampling Locations on the Site of Argonne National Laboratory

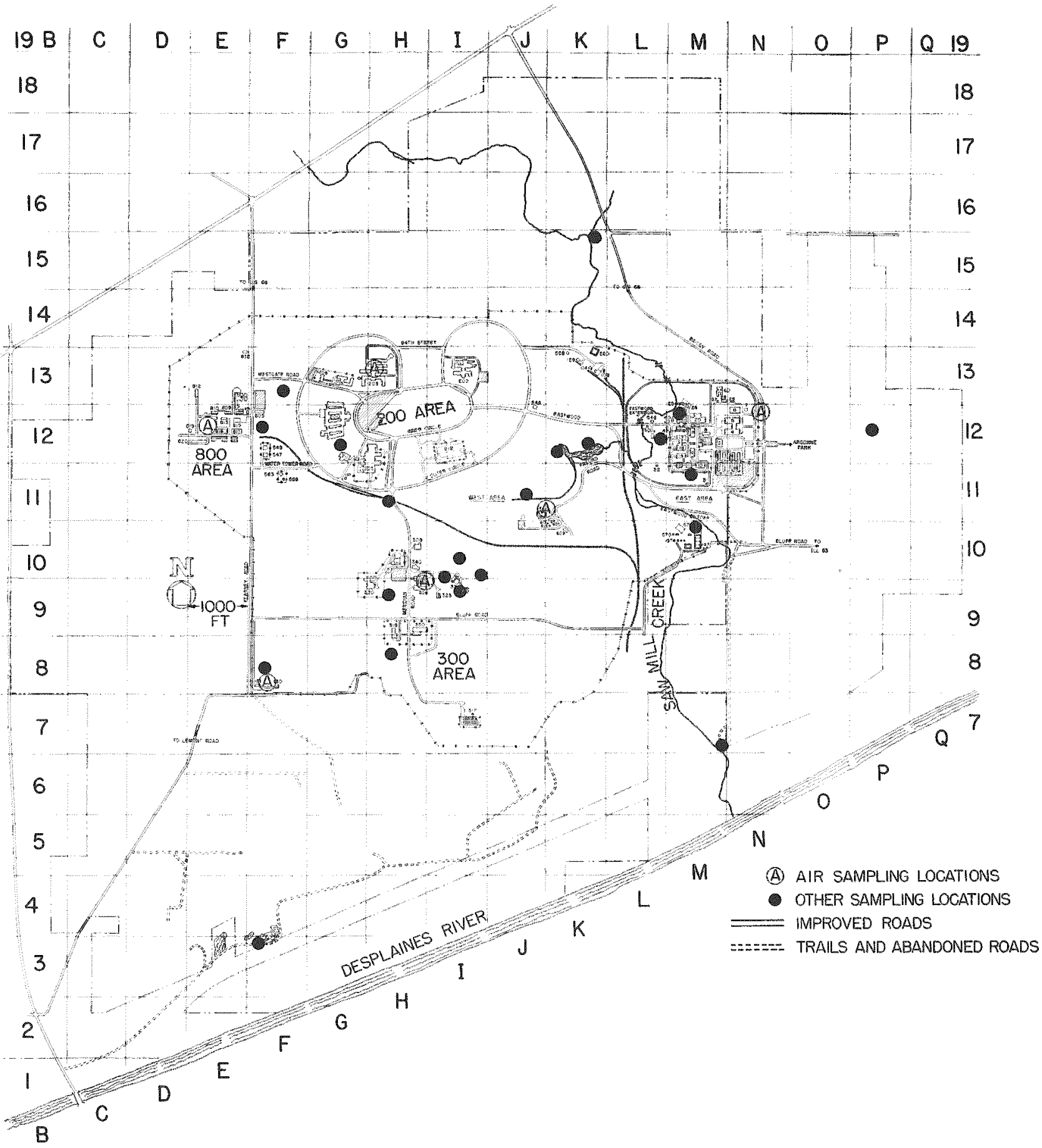


Figure 9

Sampling Locations near Argonne National Laboratory

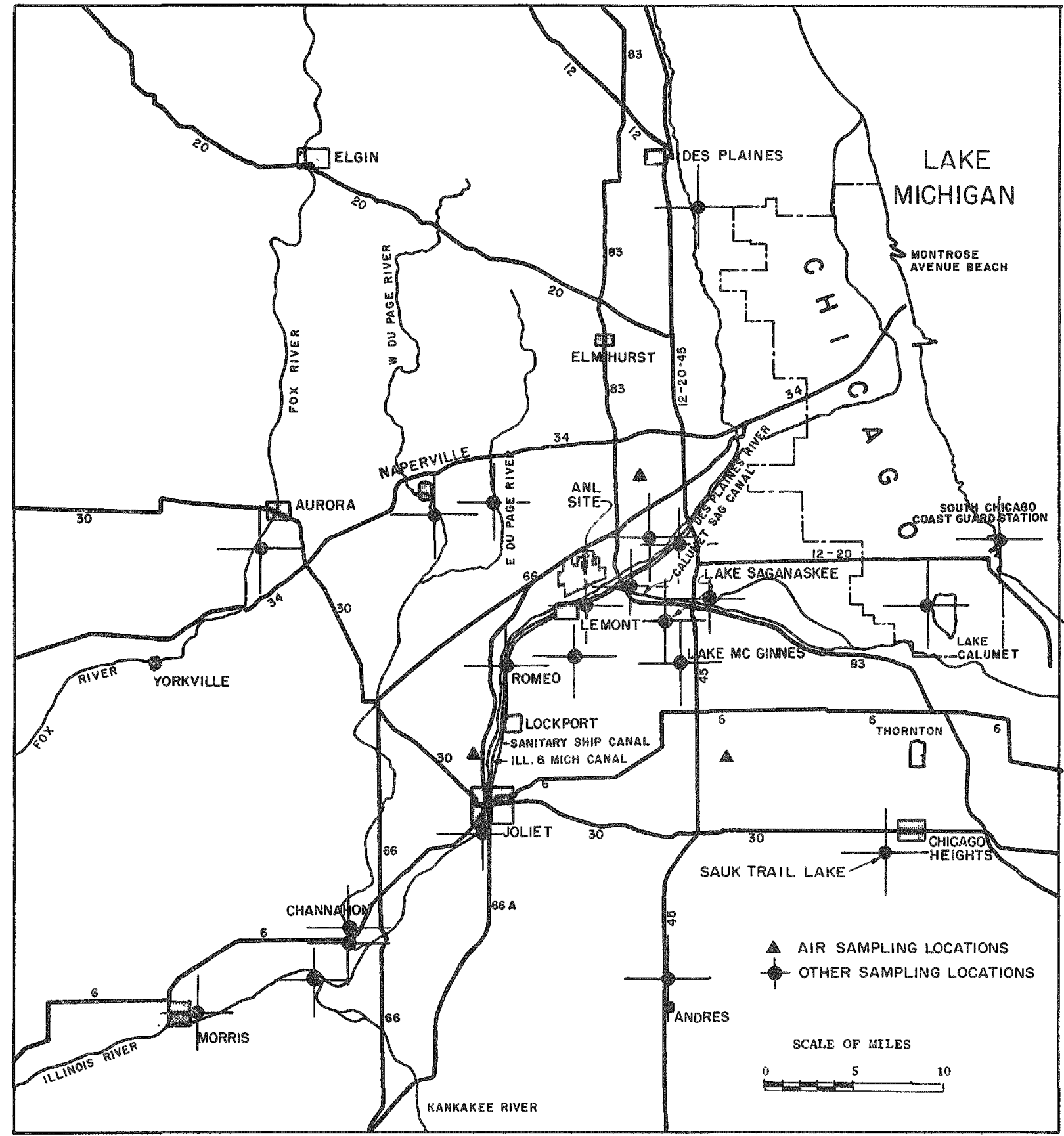


shielded beta counter. The separation procedures used were those given by Coryell and Sugarman,* suitably modified for use with the types of samples encountered. Counting rates were converted to disintegration rates by applying experimentally determined counting yields for each fission product nuclide. Concentrations of $\mathrm{Sr}^{89}$ and $\mathrm{Sr}^{90}$ and of $\mathrm{Ce}^{141}$ and $\mathrm{Ce}^{144}$ in the separated strontium and cerium fractions were calculated by an IBM -650 Computer from the total beta counting rates at various times after separation.

Uranium analyses were made with a fluorophotometer, and the results were converted to micromicrocuries, as suming the natural isotopic composition. Plutonium and thorium analyses were determined by thenoyltrifluoroacetone (TTA) extraction. After coprecipitation of both elements with cerium fluoride and dissolution of the precipitate, plutonium was reduced to the +3 state and the thorium extracted with TTA. The plutonium was then oxidized to the +4 state and extracted into TTA solution. After suitable sample preparation, the separated plutonium and thorium activities were counted in a windowless alpha counter.

Additional information on the sampling program. instrumentation, counting techniques, and analytical procedures may be found in the previous reports in this series: ANL-5069,5289,5446,5684, and 5808. The helpful assistance of John F.Ege, Jr。and John H. Pingel in the preparation of the manuscript is gratefully acknowledged.

\section{RADIOACTIVITY IN ENVIRONMENTAL SAMPLES}

\section{A. Rain}

The total alpha and beta activities in rain collected on the ANL site are given in Table II. The alpha activity that decayed during the first few days after the end of the rainfall was due to radon and thoron daughters. The alpha activity remaining after one week was due primarily to natural activities present in dust in the air or in dust blown into the rain collector. Contributions to the total beta activity were made by the sources mentioned above and also by natural potassium and fallout from nuclear detonations. Analyses for potassium and fission products have shown that the bulk of the beta activity remaining after three days was derived from fallout.

The monthly variations shown in the table may be correlated with announced detonations. Thus, the high increases in beta activity during the summer months resulted from test explosions during that period. This was confirmed from the age of the fission products as calculated by assuming a $T^{-1.2}$ decay rate. From June through most of October the calculated ages were less than thirty days, while most of the November and December samples contained fission products produced during September and October.

\footnotetext{
*"Radiochemical Studies: The Fission Products" C, D. Coryell and N. Sugarman, Eds. McGraw-Hill Book Co. Inc (New York, 1951)
} 
TABLE: II

NONVOLATILE RADIOACTIVITY IN RAINWAITER AT ANL, 1957

\begin{tabular}{|c|c|c|c|c|c|c|c|c|c|c|c|c|c|}
\hline \multirow{4}{*}{ Month } & \multirow{4}{*}{$\begin{array}{c}\text { Samples } \\
\text { Collected }\end{array}$} & \multicolumn{6}{|c|}{ Alpha Activity } & \multicolumn{6}{|c|}{ Beta Activity } \\
\hline & & \multicolumn{3}{|c|}{ After 1-Day Decay } & \multicolumn{3}{|c|}{ After 1 - Week Decay } & \multicolumn{3}{|c|}{ After 1-Day Decay } & \multicolumn{3}{|c|}{ After 1-Week Decay } \\
\hline & & \multirow{2}{*}{$\begin{array}{l}\text { Samples } \\
\text { Counted }\end{array}$} & \multicolumn{2}{|c|}{$\mu \mu \mathrm{c} /$ /1ter } & \multirow{2}{*}{$\begin{array}{l}\text { Samples } \\
\text { Counted }\end{array}$} & \multicolumn{2}{|c|}{ Halliter } & \multirow{2}{*}{$\begin{array}{l}\text { Samples } \\
\text { Counted }\end{array}$} & \multicolumn{2}{|c|}{$100 \mu \mu \mathrm{c} / 1 \mathrm{it}+\mathrm{s}$} & \multirow{2}{*}{$\begin{array}{l}\text { Samples } \\
\text { Counted }\end{array}$} & \multicolumn{2}{|c|}{$100 \mu \mu \mathrm{c} / \mathrm{Liter}$} \\
\hline & & & Max. & Av. & & Max. & Av. & & Max. & Av. & & $\operatorname{Max}$ & $\mathrm{Av}$ \\
\hline January & 11 & 9 & 31.0 & 11.7 & 11 & 28.0 & 10.9 & 9 & 33.7 & 138 & 11 & 33.2 & 14.0 \\
\hline February & 8 & 7 & 10.1 & 4.7 & 8 & 9.6 & 3.8 & 7 & 14.5 & 78 & 8 & 13.4 & 6.4 \\
\hline March & 5 & 5 & 17.9 & 8.0 & 5 & 38 & 19 & 5 & 27.4 & 10.5 & 5 & 160 & 7.3 \\
\hline April. & 19 & 15 & 22.9 & 5.3 & 19 & 10.1 & 17 & 15 & 415 & 17.2 & 19 & 361 & 95 \\
\hline May & 12 & 11 & 21.0 & 41 & 11 & 180 & 30 & 11 & 34.4 & 8.1 & 11 & 329 & 75 \\
\hline June & 18 & 17 & 790 & 123 & 18 & 110 & 21 & 17 & 613 & 13.6 & 18 & 466 & 10.7 \\
\hline July & 7 & 7 & 228 & 5.5 & 7 & 63 & 18 & 7 & 192 & 70 & 7 & 135 & 56 \\
\hline August & 12 & 7 & 400 & 138 & 11 & 369 & 9.4 & 12 & 513 & 106 & 12 & 350 & 79 \\
\hline September & 7 & 7 & 74 & 28 & 7 & 41 & 16 & 7 & 195 & 55 & 7 & 138 & 39 \\
\hline October & 8 & 8 & 142 & 2.8 & 8 & 80 & 20 & 8 & 826 & 20.5 & 8 & 660 & 16.0 \\
\hline November & 8 & 7 & 34 & 15 & 8 & 15 & 06 & 8 & 56 & 26 & 8 & 50 & 13 \\
\hline December & 7 & 6 & 8.9 & 33 & 8 & 89 & 2.5 & 6 & 113 & 63 & 7 & 10.6 & 5.1 \\
\hline
\end{tabular}

* Two successive portions were collccted from heavy rains and are considered as separate samples Each portion was equivalent to 0.1 inch of rain.

In general, high concentrations of beta activity were associated with recent detonation dates. It should be pointed out that an accurate detonation date can be obtained from the decay rate only if most of the activity carried down by a rainfall was derived from one explosion, or from a very few explosions occurring within a short time interval.

The increase in beta activity during the summer and fall months was also noted in other types of samples, so that beta-activity concentrations in rain were useful in interpreting beta activities in other samples. The general trend in fallout activity in rainfall may be seen from Figure 10. Except for a few months of very high activity during each year there has been a general increase in the past two years from a median value of about $200 \mu \mu \mathrm{c} / 1$ to about $700 \mu \mu \mathrm{c} / 1$. This was probably the result of bomb testing being conducted over a larger fraction of the year during 1956 and 1957 . Since a small fraction of the rainfalls carry down most of the activity during any one year, the total amount of beta activity carried down each year has not increasedas the median value has. During 1955 and 1957 the total activity, as calculated from the one-week activities in each sample, was about 1200 millicuries per square mile. For 1953, 1954, and 1956 the corresponding value was about 600 , while in 1952 about $11,000 \mathrm{mc} / \mathrm{mi}^{2}$ were found in the rains. About $95 \%$ of the latter amount was found in the samples collected in June, 1952.

\section{B. Air Filters}

Air-borne particulate matter was sampled at five locations on the Laboratory site, and, beginning in May, at three locations off the Laboratory site. To collect the sample, air was filtered through HollingsworthVose No. 70 filter paper using a Filter-Queen Vacuum Cleaner modified to draw air through the paper, or a Sutorbilt Air Sampler. A rotameter was used to measure the air flow; the Filter-Queen sampler operated at 15 cubic 
Figure 10

AVERAGE MONTHLY BETA ACTIVITY IN RAINWATER, 1952-57

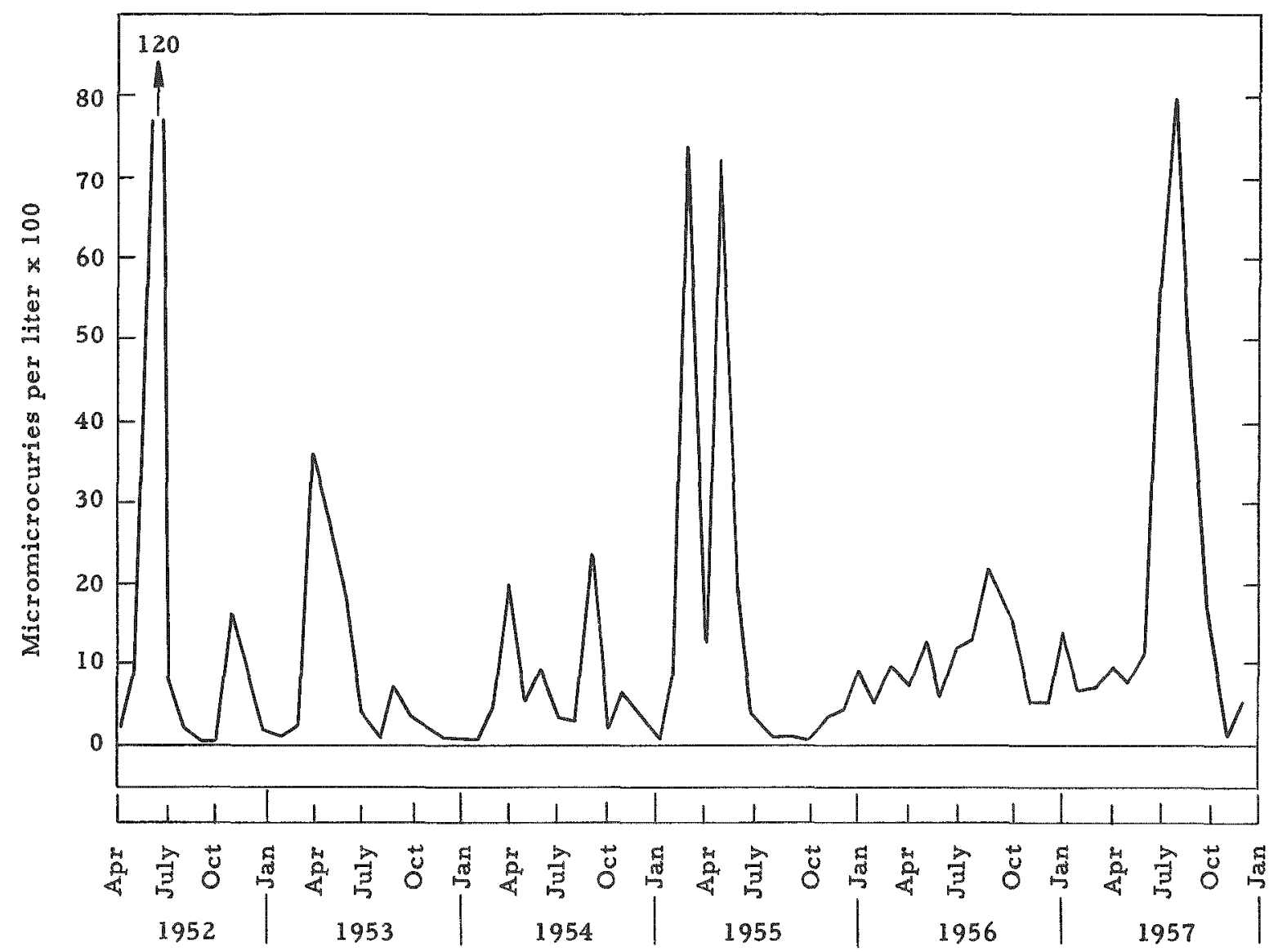

meters per hour and the Sutorbilt sampler at 30 cubic meters per hour. Twenty-four hour samples were collected with one sampler on the Labora tory site to record short-term changes in air activity. The remaining onsite samplers, and all off-site samplers, were operated for a one-week sampling period. The instruments were operated continuously except for equipment failures and the few minutes required to change filter papers. After the sampling period the paper was sprayed with a solution of polystyrene in ethylene dichloride to fix the particulate matter on the paper: the thickness of the polystyrene layer was 50 micrograms per $\mathrm{cm}^{2}$. The area of the paper was $200 \mathrm{~cm}^{2}$. A circle, 2 inches in diameter $\left(20 \mathrm{~cm}^{2}\right)$, was cut from the center of the paper, mounted on a stainless steel counting planchet, and counted for alpha and beta activities in a nylon-window proportional counter. Monthly summaries of weekly air-filter samples collected from 1953 through 1957 and daily samples collected in 1957 are given in Tables III to VIII. The results for daily samples collected from 1953-1956 were given in ANL-5808. The results given in the tables were calculated from the counting rates three and seven days after the end of 
TABLE III

TOTAL ALPHA AND BETA ACTIVITIES IN WEEIIY AIR FILTER SAMPLES COLLECTED ON ANL SITE, 1953

\begin{tabular}{|c|c|c|c|c|c|c|c|c|c|c|}
\hline \multirow{3}{*}{ Month } & \multicolumn{5}{|c|}{$\mu \mu \mathrm{c} / \mathrm{M}^{3}$ after $3-$ Day Decay } & \multicolumn{5}{|c|}{$\mu \mu \mathrm{c} / \mathrm{M}^{3}$ after 7 -Day Decay } \\
\hline & \multirow{2}{*}{$\begin{array}{c}\text { No. of } \\
\text { Samples }\end{array}$} & \multicolumn{2}{|c|}{ Alpha Act. } & \multicolumn{2}{|c|}{ Beta Act. } & \multirow{2}{*}{$\begin{array}{c}\text { No. of } \\
\text { Samples }\end{array}$} & \multicolumn{2}{|c|}{ Alpha Act. } & \multicolumn{2}{|c|}{ Beta Act. } \\
\hline & & Max. & Av. & Max. & Av. & & Max. & Av. & Max. & Av. \\
\hline January & 0 & - & - & - & - & 15 & 0.007 & 0.004 & 0.13 & 0.08 \\
\hline February & 3 & 0.009 & 0.007 & 0.11 & 0.09 & 12 & .007 & .004 & 0.09 & 0.06 \\
\hline March & 12 & .009 & .007 & 3.8 & 0.8 & 12 & .007 & .005 & 2.3 & 0.6 \\
\hline April & 11 & .010 & .007 & 1.1 & 0.5 & 11 & .006 & .005 & 0.82 & 0.41 \\
\hline May & 14 & .030 & .011 & 4.2 & 1.9 & 15 & .024 & .007 & 2.7 & 1.3 \\
\hline June & 12 & .036 & .013 & 28.7 & 5.6 & 12 & .036 & .007 & 23.3 & 4.5 \\
\hline July & 9 & .015 & .009 & 2.1 & 1.6 & 11 & .006 & .004 & 2.0 & 1.3 \\
\hline August & 15 & .024 & .012 & 0.92 & 0.65 & 15 & .014 & .005 & 0.84 & 0.60 \\
\hline September & 11 & .020 & .016 & 2.8 & 2.0 & 12 & .011 & .006 & 2.4 & 1.7 \\
\hline October & 13 & .038 & .018 & 1.9 & 0.8 & 13 & .017 & .008 & 1.7 & 0.7 \\
\hline November & 10 & .025 & .018 & 0.48 & 0.37 & 10 & .010 & .007 & 0.44 & 0.33 \\
\hline December & 4 & .006 & .005 & 0.20 & 0.15 & 8 & .011 & .004 & 0.21 & 0.14 \\
\hline Average & & \multicolumn{2}{|c|}{0.010} & \multicolumn{2}{|c|}{1.2} & & \multicolumn{2}{|c|}{0.006} & \multicolumn{2}{|c|}{1.0} \\
\hline
\end{tabular}

TABLE IV

TOTAL ALPHA AND BETA ACTIVITIES IN WEEKLY AIR FILTER SAMPLES COLLECTED ON ANL SITE, 1954

\begin{tabular}{|c|c|c|c|c|c|c|c|c|c|c|}
\hline \multirow{3}{*}{ Month } & \multicolumn{5}{|c|}{$\mu \mu \mathrm{c} / \mathrm{M}^{3}$ after 3-Day Decay } & \multicolumn{5}{|c|}{$\mu \mu_{\mathrm{c}} / \mathrm{M}^{3}$ after 7-Day Decay } \\
\hline & \multirow{2}{*}{$\begin{array}{c}\text { No. of } \\
\text { Samples }\end{array}$} & \multicolumn{2}{|c|}{ Alpha Act. } & \multicolumn{2}{|c|}{ Beta Act. } & \multirow{2}{*}{$\begin{array}{c}\text { No. of } \\
\text { Samples }\end{array}$} & \multicolumn{2}{|c|}{ Alpha Act. } & \multicolumn{2}{|c|}{ Beta Act. } \\
\hline & & Max. & Av. & $\operatorname{Max}$. & Av. & & Max. & Av. & Max. & Av. \\
\hline January & 4 & 0.012 & 0.008 & 0.11 & 0.10 & 4 & 0.007 & 0.006 & 0.11 & 0.10 \\
\hline February & 10 & .143 & .019 & .39 & .12 & 10 & .005 & .004 & .12 & .09 \\
\hline March & 8 & .009 & .005 & .69 & .31 & 9 & .006 & .004 & .52 & .24 \\
\hline April & 12 & .013 & .011 & .57 & .30 & 12 & .008 & .005 & .50 & .26 \\
\hline May & 10 & .032 & .012 & .34 & .20 & 10 & .014 & .006 & .32 & .16 \\
\hline June & 11 & .011 & .010 & .79 & .35 & 12 & .004 & .003 & .72 & .30 \\
\hline July & 11 & .013 & .010 & .46 & .27 & 11 & .007 & .004 & .43 & .24 \\
\hline August & 10 & .010 & .007 & .40 & .33 & 11 & .008 & .004 & .40 & .30 \\
\hline September & 13 & .025 & .015 & 2.3 & .5 & 12 & .006 & .003 & .50 & .26 \\
\hline October & 8 & .019 & .008 & 2.0 & .7 & 14 & .012 & .005 & 1.2 & .6 \\
\hline November & 17 & .029 & .011 & 2.2 & 1.2 & 17 & .013 & .007 & 1.8 & 1.0 \\
\hline December & 15 & .011 & .005 & 0.93 & 0.61 & 15 & .006 & .003 & 0.87 & 0.56 \\
\hline Average & & \multicolumn{2}{|c|}{0.010} & \multicolumn{2}{|c|}{0.4} & & \multicolumn{2}{|c|}{0.005} & \multicolumn{2}{|c|}{0.3} \\
\hline
\end{tabular}


TABLE V

TOTAL ALPHA AND BETA ACTIVITIES IN WEEILI $Y$ AIR FILTER SAMPLES COLLECTED ON ANL SITE, 1955

\begin{tabular}{|c|c|c|c|c|c|c|c|c|c|c|}
\hline \multirow{3}{*}{ Month } & \multicolumn{5}{|c|}{$\mu \mu \mathrm{c} / \mathrm{M}^{3}$ after 3-Day Decay } & \multicolumn{5}{|c|}{$\mu_{\mathrm{c}} / \mathrm{M}^{3}$ aftex 7-Day Decay } \\
\hline & \multirow{2}{*}{$\begin{array}{c}\text { No. of } \\
\text { Samples }\end{array}$} & \multicolumn{2}{|c|}{ Alpha Act. } & \multicolumn{2}{|c|}{ Beta Act. } & \multirow{2}{*}{$\begin{array}{c}\text { No. of } \\
\text { Samples }\end{array}$} & \multicolumn{2}{|c|}{ Alpha Act. } & \multicolumn{2}{|c|}{ Beta Act. } \\
\hline & & Max. & $\mathrm{Av}$. & Max. & $\mathrm{Av}$. & & Max. & Av. & Max. & Av. \\
\hline January & 13 & 0.009 & 0.005 & 0.47 & 0.29 & 10 & 0.006 & 0.005 & 0.42 & 0.28 \\
\hline February & 12 & .013 & .006 & 2.1 & 0.6 & 13 & .013 & .005 & 1.3 & 0.7 \\
\hline March & 19 & .020 & .007 & 3.8 & 1.2 & 19 & .007 & .005 & 2.4 & 0.9 \\
\hline April & 18 & .020 & .008 & 2.0 & 1.3 & 17 & .009 & .005 & 1.8 & 1.0 \\
\hline May & 22 & .019 & .009 & 12.2 & 3.6 & 19 & .013 & .004 & 9.2 & 2.7 \\
\hline June & 18 & .023 & .008 & 2.3 & 1.0 & 18 & .007 & .004 & 2.2 & 0.9 \\
\hline July & 19 & .031 & .011 & 1.3 & 0.5 & 17 & .023 & .006 & 0.88 & 0.42 \\
\hline August & 17 & .019 & .009 & 0.42 & 0.29 & 18 & .008 & .004 & 0.42 & 0.25 \\
\hline September & 13 & .021 & .007 & 0.32 & 0.19 & 14 & .006 & .003 & 0.30 & 0.18 \\
\hline October & 9 & .019 & .006 & 0.17 & 0.12 & 15 & .009 & .003 & 0.17 & 0.11 \\
\hline November & 15 & .007 & .004 & 1.0 & 0.3 & 16 & .006 & .003 & 0.71 & 0.28 \\
\hline December & 18 & .010 & .005 & 1.7 & 0.9 & 15 & .006 & .004 & 1.6 & 0.8 \\
\hline Average & & \multicolumn{2}{|c|}{0.007} & \multicolumn{2}{|c|}{0.8} & & \multicolumn{2}{|c|}{0.004} & \multicolumn{2}{|c|}{0.7} \\
\hline
\end{tabular}

TABLE VI

TOTAL ALPHA AND BETA ACTIVITIES IN WEEKLY AIR FILTER

SAMPLES COLLECTED ON ANL SITE, 1956

\begin{tabular}{|c|c|c|c|c|c|c|c|c|c|c|}
\hline \multirow{3}{*}{ Month } & \multicolumn{5}{|c|}{$\mu \mu \mathrm{c} / \mathrm{M}^{3}$ after 3-Day Decay } & \multicolumn{5}{|c|}{$\mu \mu_{\mathrm{c}} / \mathrm{M}^{3}$ after 7 -Day Decay } \\
\hline & \multirow{2}{*}{$\begin{array}{c}\text { No. of } \\
\text { Samples }\end{array}$} & \multicolumn{2}{|c|}{ Alpha Act. } & \multicolumn{2}{|c|}{ Beta Act. } & \multirow{2}{*}{$\begin{array}{c}\text { No. of } \\
\text { Samples }\end{array}$} & \multicolumn{2}{|c|}{ Alpha Act. } & \multicolumn{2}{|c|}{ Beta Act. } \\
\hline & & Max. & Av. & Max. & Av. & & Max. & Av. & Max. & Av. \\
\hline January & 15 & 0.012 & 0.006 & 2.3 & 1.7 & 12 & 0.014 & 0.005 & 2.1 & 1.6 \\
\hline February & 13 & .006 & .004 & 1.5 & 0.9 & 12 & .006 & .005 & 1.4 & 0.9 \\
\hline March & 16 & .010 & .006 & 1.3 & 1.0 & 15 & .007 & .005 & 1.2 & 0.9 \\
\hline April & 12 & .016 & .009 & 2.2 & 1.4 & 8 & .008 & .005 & 2.2 & 1.2 \\
\hline May & 13 & .030 & .008 & 1.5 & 1.0 & 12 & .025 & .006 & 1.4 & 1.0 \\
\hline June & 6 & .025 & .009 & 1.4 & 0.8 & 6 & .005 & .003 & 1.3 & 0.8 \\
\hline July & 10 & .038 & .017 & 1.1 & 0.7 & 11 & .021 & .008 & 1.0 & 0.6 \\
\hline August & 14 & .029 & .010 & 1.5 & 0.9 & 13 & .028 & .005 & 1.4 & 0.8 \\
\hline September & 14 & .033 & .016 & 7.9 & 3.1 & 14 & .010 & .005 & 6.1 & 2.5 \\
\hline October & 12 & .033 & .016 & 3.5 & 2.0 & 15 & .013 & .008 & 3.6 & 2.1 \\
\hline November & 14 & .026 & .012 & 1.3 & 0.9 & 15 & .010 & .005 & 1.1 & 0.8 \\
\hline December & 12 & .022 & .007 & 1.7 & 1.0 & 16 & .021 & .006 & 1.5 & 0.8 \\
\hline Average & & \multicolumn{2}{|c|}{0.010} & \multicolumn{2}{|c|}{1.3} & & \multicolumn{2}{|c|}{0.006} & \multicolumn{2}{|c|}{1.2} \\
\hline
\end{tabular}


TABLE VII

TOTAL ALPHA AND BETA ACTIVITIES IN WEEKLY AIR

FILTER SAMPLES, 1957

\begin{tabular}{|c|c|c|c|c|c|c|c|c|c|c|}
\hline \multirow{3}{*}{ Month } & \multirow{3}{*}{ Location } & \multirow{3}{*}{$\begin{array}{l}\text { No. of } \\
\text { Samples }\end{array}$} & \multicolumn{4}{|c|}{$\mu \mu \mathrm{c} / \mathrm{M}^{3}$ after $3-$ Day Decay } & \multicolumn{4}{|c|}{$\mu \mu \mathrm{c} / \mathrm{M}^{3}$ after 7 -Day Decay } \\
\hline & & & \multicolumn{2}{|c|}{ Alpha Act. } & \multicolumn{2}{|c|}{ Beta Act. } & \multicolumn{2}{|c|}{ Alpha Act. } & \multicolumn{2}{|c|}{ Beta Act. } \\
\hline & & & Max. & Av. & Max. & Av. & Max. & Av. & Max. & Av. \\
\hline January & ANL & 17 & 0.012 & 0.005 & 1.3 & 1.0 & 0.008 & 0.004 & 1.2 & 0.9 \\
\hline February & ANL & 7 & .010 & .007 & 1.4 & 1.1 & .008 & .005 & 1.3 & 1.1 \\
\hline March & ANL & 14 & .012 & .006 & 2.5 & 1.4 & .007 & .005 & 2.2 & 1.3 \\
\hline April & ANL & 18 & .021 & .008 & 2.7 & 1.6 & .008 & .005 & 2.7 & 1.6 \\
\hline May & $\begin{array}{l}\text { ANL } \\
\text { Near ANL }\end{array}$ & $\begin{array}{r}18 \\
4\end{array}$ & $\begin{array}{l}.017 \\
.007\end{array}$ & $\begin{array}{l}.008 \\
.005\end{array}$ & $\begin{array}{l}3.4 \\
1.7\end{array}$ & $\begin{array}{l}2.0 \\
1.1\end{array}$ & $\begin{array}{l}.010 \\
.006\end{array}$ & $\begin{array}{l}.006 \\
.004\end{array}$ & $\begin{array}{l}3.3 \\
1.6\end{array}$ & $\begin{array}{l}1.9 \\
1.1\end{array}$ \\
\hline June & $\begin{array}{l}\text { ANL } \\
\text { Near ANL }\end{array}$ & $\begin{array}{r}10 \\
4\end{array}$ & $\begin{array}{l}.016 \\
.009\end{array}$ & $\begin{array}{l}.009 \\
.006\end{array}$ & $\begin{array}{l}3.0 \\
1.5\end{array}$ & $\begin{array}{l}1.9 \\
1.3\end{array}$ & $\begin{array}{l}.007 \\
.005\end{array}$ & $\begin{array}{l}.004 \\
.004\end{array}$ & $\begin{array}{l}2.8 \\
1.4\end{array}$ & $\begin{array}{l}1.6 \\
1.3\end{array}$ \\
\hline July & $\begin{array}{l}\text { ANL } \\
\text { Near ANL }\end{array}$ & $\begin{array}{r}13 \\
8\end{array}$ & $\begin{array}{l}.022 \\
.030\end{array}$ & $\begin{array}{l}.010 \\
.015\end{array}$ & $\begin{array}{l}8.9 \\
2.8\end{array}$ & $\begin{array}{l}3.1 \\
2.0\end{array}$ & $\begin{array}{l}.011 \\
.005\end{array}$ & $\begin{array}{l}.005 \\
.004\end{array}$ & $\begin{array}{l}2.8 \\
2.4\end{array}$ & $\begin{array}{l}1.9 \\
1.5\end{array}$ \\
\hline August & $\begin{array}{l}\text { ANL } \\
\text { Near ANL }\end{array}$ & $\begin{array}{r}16 \\
4\end{array}$ & $\begin{array}{l}.042 \\
.016\end{array}$ & $\begin{array}{l}.016 \\
.010\end{array}$ & $\begin{array}{r}11.1 \\
9.3\end{array}$ & $\begin{array}{l}3.6 \\
5.3\end{array}$ & $\begin{array}{l}.015 \\
.017\end{array}$ & $\begin{array}{l}.005 \\
.010\end{array}$ & $\begin{array}{l}7.9 \\
7.1\end{array}$ & $\begin{array}{l}3.0 \\
3.9\end{array}$ \\
\hline September & $\begin{array}{l}\text { ANL } \\
\text { Near ANL }\end{array}$ & $\begin{array}{r}10 \\
8\end{array}$ & $\begin{array}{l}.016 \\
.006\end{array}$ & $\begin{array}{l}.008 \\
.004\end{array}$ & $\begin{array}{l}8.5 \\
4.9\end{array}$ & $\begin{array}{l}4.1 \\
2.6\end{array}$ & $\begin{array}{l}.006 \\
.004\end{array}$ & $\begin{array}{l}.003 \\
.003\end{array}$ & $\begin{array}{l}6.1 \\
5.7\end{array}$ & $\begin{array}{l}3.1 \\
2.3\end{array}$ \\
\hline October & $\begin{array}{l}\text { ANL } \\
\text { Near ANL }\end{array}$ & $\begin{array}{r}13 \\
6\end{array}$ & $\begin{array}{l}.043 \\
.026\end{array}$ & $\begin{array}{l}.010 \\
.008\end{array}$ & $\begin{array}{l}7.7 \\
2.7\end{array}$ & $\begin{array}{l}3.0 \\
1.7\end{array}$ & $\begin{array}{l}.009 \\
.005\end{array}$ & $\begin{array}{l}.003 \\
.003\end{array}$ & $\begin{array}{l}6.3 \\
2.5\end{array}$ & $\begin{array}{l}2.4 \\
1.5\end{array}$ \\
\hline November & ANL & 10 & .018 & .006 & 3.0 & 1.3 & .006 & .003 & 3.0 & 1.2 \\
\hline December & $\begin{array}{l}\text { ANL } \\
\text { Near ANL }\end{array}$ & $\begin{array}{l}7 \\
7\end{array}$ & $\begin{array}{l}.007 \\
.007\end{array}$ & $\begin{array}{l}.005 \\
.003\end{array}$ & $\begin{array}{l}2.0 \\
1.6\end{array}$ & $\begin{array}{l}1.0 \\
1.0\end{array}$ & $\begin{array}{l}.006 \\
.006\end{array}$ & $\begin{array}{l}.004 \\
.003\end{array}$ & $\begin{array}{l}1.5 \\
1.6\end{array}$ & $\begin{array}{l}0.9 \\
0.8\end{array}$ \\
\hline Average & $\begin{array}{l}\text { ANL } \\
\text { Near ANL }\end{array}$ & & & $\begin{array}{l}008 \\
009)^{*} \\
007\end{array}$ & $\begin{array}{r}2.1 \\
(2.7 \\
2.1\end{array}$ & & $\begin{array}{r}0.0 \\
0.0 \\
0.0\end{array}$ & $\begin{array}{l}5 \\
4)^{*} \\
4\end{array}$ & $\begin{array}{r}1.7 \\
(2.2 \\
1.7\end{array}$ & \\
\hline
\end{tabular}

*ANL samples, May through October and December only.

the filtering period for weekly samples and after three days for daily samples. The factors used to convert counting rates to disintegration rates, and the validity of the results, are discussed in ANL-5808. The weekly samples were also counted during the first day after the sampling period, and the radon and thoron concentrations obtained from these counting rates will be reported at a later date.

The activities remaining after three days were due primarily to fission products (for most of the beta activity) and to long-lived naturally occurring radioactive nuclides (for the alpha activity and a small fraction of the beta activity). The beta activities showed periodic fluctuations, with increases observed during periods of nuclear detonations. A comparison 
TABLE VIII

LONG-LIVED RADIOACTIVITIES IN 24-HOUR AIR-FILTER SAMPLES COLLECTED ON

ANL SITE, 1957

\begin{tabular}{|l|c|c|c|c|c|}
\hline \multirow{2}{*}{ Month } & \multirow{2}{*}{$\begin{array}{c}\text { No. of } \\
\text { Samples }\end{array}$} & \multicolumn{2}{c|}{$\begin{array}{c}\text { Alpha. Activity } \\
\left(\mu \mu \mathrm{c} / \mathrm{M}^{3}\right)\end{array}$} & \multicolumn{2}{c|}{$\begin{array}{c}\text { Beta Activity } \\
\left(\mu \mu \mathrm{c} / \mathrm{M}^{3}\right)\end{array}$} \\
\cline { 3 - 6 } & & Max. & Av. & Max. & Av. \\
\hline January & 25 & 0.020 & 0.009 & 2.2 & 1.1 \\
February & 25 & 0.047 & 0.008 & 2.7 & 1.2 \\
March & 31 & 0.068 & 0.016 & 4.1 & 1.5 \\
April & 27 & 0.068 & 0.018 & 2.8 & 1.2 \\
May & 29 & 0.097 & 0.016 & 6.0 & 2.1 \\
June & 30 & 0.071 & 0.022 & 3.4 & 1.8 \\
July & 23 & 0.092 & 0.038 & 25.3 & 3.5 \\
August & 30 & 0.451 & 0.044 & 15.5 & 3.4 \\
September & 27 & 0.092 & 0.021 & 12.9 & 3.1 \\
October & 21 & 0.050 & 0.029 & 12.7 & 2.5 \\
November & 28 & 0.032 & 0.013 & 9.2 & 1.4 \\
December & 28 & 0.090 & 0.022 & 4.1 & 1.1 \\
\hline Average & & \multicolumn{2}{|c|}{0.021} & \multicolumn{2}{|c|}{2.0} \\
\hline
\end{tabular}

of the third and seventh-day activities indicated that these increases were associated with shorter-lived activities than were present during periods of lesser fallout. The long-term trend for fission product activity in air may be seen from the summary in Table IX.

TABLE IX

ANNUAL AVERAGE OF "THIRD DAY" BETA ACTIVITY IN AIR ON ANL SITE

\begin{tabular}{|c|c|c|c|c|}
\hline \multirow{2}{*}{ Year } & \multicolumn{2}{|c|}{ Weekly Samples } & \multicolumn{2}{c|}{ Daily Samples } \\
\cline { 2 - 5 } & $\begin{array}{c}\text { No. of } \\
\text { Samples }\end{array}$ & $\left(\mu \mu \mathrm{c} / \mathrm{M}^{3}\right)$ & $\begin{array}{c}\text { No. of } \\
\text { Samples }\end{array}$ & $\left(\mu \mu \mathrm{c} / \mathrm{M}^{3}\right)$ \\
\hline 1953 & 114 & 1.2 & 322 & 1.4 \\
1954 & 129 & 0.4 & 345 & 0.5 \\
1955 & 193 & 0.8 & 345 & 1.0 \\
1956 & 151 & 1.3 & 345 & 1.6 \\
1957 & 153 & 2.1 & 324 & 2.0 \\
\hline
\end{tabular}

The beta activity has increased slowly but consistently from 1954 to 1957, following a decrease from 1953 to 1954 . The increase in the average in recent years has not been accompanied by an increase in the maximum values during periods of bomb testing, but by an increase in the median value. For example, the highest individual result, $127 \mu \mu \mathrm{c} / \mathrm{M}^{3}$, was obtained in May, 1953, while the maximum value during 1957 was only 
$25.3 \mu \mu \mathrm{c} / \mathrm{M}^{3}$. However, in 1953 monthly averages less than $1 \mu \mu \mathrm{c} / \mathrm{M}^{3}$ were found during seven months, while in 1957 the minimum monthly average was $1.1 \mu \mu \mathrm{c} / \mathrm{M}^{3}$. The reason for this trend is probably the same as that given for a similar trend in rain. That is, during recent years, testing has been conducted over a larger fraction of the year.

There was good agreement between the beta activity in the weekly and daily air-filter samples during periods of low fallout. However, when the concentration of short-lived fission products increased, differences in beta activities between the two types of samples were noted. At such times the collection of a few high-activity particles on a filter paper will have a large effect on the results. For the weelly air filters, the time during the sampling period when such particles are collected becomes very important, and quantitative agreement between the two types of samples cannot be expected. The daily samples probably give a more accurate picture of the total amount of fallout. The collection of weekly samples, how ever, permits the analysis of a larger number of samples with inexpensive air samplers and fewer counters than would otherwise be possible. A large number of locations can be monitored in this way, and, since the papers are changed on different days at the various locations, the times at which changes in activity occur can still be obtained. In addition, the weekly samples will be useful in determining the concentrations of long-lived fission products in air. There was a consistent difference in long-lived alpha activity between the weekly and daily air filters on the site as a result of differences in sample thickness for which adequate corrections had not been made. However, each type of sample yields consistent results and comparison may be made between samples collected for the same period of time. The average third-day alpha activity in the daily samples was 0.02 in 1957, and 0.03 from 1953 - 1956: the corresponding results for the weekly samples ranged from 0,007 to 0.010 . Thus, the alpha activities have remained quite constant, and consistent for each collection period, and do not vary with the beta activities.

Samplers were located on the periphery of the site as well as near buildings containing large amounts of radioactive materials. One sampler was located between the EBWR and CP-5 reactors. No significant or consistent differences between samples obtained at the various on-site locations could be detected. Gaseous contaminants, including tritium and tritiated water, were not collected by these samples. A program is underway to determine these materials in air.

In May, samples collection was begun at locations off the Laboratory site. Samples were collected at Stateville Penitentiary, 10 miles southwest of the ANL site: Suburban Cook County Tuberculosis Sanitarium near Hinsdale, 6 miles northweat of the site; and Tinley Park State Hospital, 13 miles southeast of the site. These locations were obtained through the cooperation of the Illinois Department of Public Health. As shown in 
Table VII, the differences in beta activity between the off-site and on-site samples varied in magnitude and direction, averaging $0.6 \mu \mu \mathrm{c} / \mathrm{M}^{3}$ higher on the on-site. Variations in the amount of fallout collected at different locations may have contributed to these differences, since they were greater during periods of increased fallout and were greater after threeday decay than after seven-day decay. Another important variable was the type of air sampler used. Because of their greater mechanical reliability and durability. Sutorbilt Air samplers were used off the ANL site: Filter-Queen samplers were used on the site. Since the Sutorbilt samplers operate at twice the flow rate of the Filter-Queen instruments, a laxger amount of dust was collected by the former. The same correction factors were used for samples collected by both instruments, so errors tending to give lower results with the Sutorbilt samplers were introduced in this way. The quantitative effects of these variables in sample collection are not known, but a study is underway to evaluate them. It is intended to replace the Filter-Queen samplers as the Sutorbilt samplers become available. The average alpha activity was approximately the same both off and on the Laboratory site, although some individual samples collected from different locations during the same time interval differed by factors of two to three. Since the amount of long-lived alpha activity collected on the filter paper is a function of the amount of dust in the air, differences due only to location may be expected.

From June through December air samplers were operated simultaneously at the top of the 120-foot high Meteorology Tower on the site and on the ground approximately 100 yards from the tower. The air intake on the latter sampler was located three feet from the ground. The tower samples were slightly, but consistently, higher than the sample collected near the ground. The averages for the tower samples were $0.005 \mu \mu \mathrm{c} \alpha / \mathrm{M}^{3}$ and $0.68 \mu \mu \mathrm{c} \beta / \mathrm{M}^{3}$ : the corresponding values for the ground samples were $0.003 \mu \mu \mathrm{c} \alpha / \mathrm{M}^{3}$ and $0.53 \mu \mu \mathrm{c} \beta / \mathrm{M}^{3}$. The same self absorption factors were used for both types of samples and, if the concentrations of particulate radioactivity were essentially the same at both heights, the additional self-absorption in the ground samples, due to the larger amount of material collected, would account for this difference.

\section{Water}

\section{Sawmill Creek and Des Plaines River}

Sawmill Creek was sampled above the Laboratory site (at square $15 \mathrm{~K}$ in Figure 8) and below the Argonne waste water outfall (square $7 \mathrm{M}$ ). Monthly summaries of the alpha activities found at both locations are given in Table $\mathrm{X}$. The water collected below the outfall contained an average of $12 \mu \mu \mathrm{c} \alpha / 1$, or about $9 \mu \mu \mathrm{c} / 1$ more than from above the site. This difference is attributed to activity added to the Creek in Argonne waste water and amounts to $9 \%$ of the maximum permissible concentration (MPL) 
TABLE X

NONVOLATILE ALPHA ACTIVITIES IN SAWMILL CREEK WATER, 1957

\begin{tabular}{|c|c|c|c|c|c|c|c|c|c|c|c|c|c|c|c|}
\hline \multirow{4}{*}{ Month } & \multirow{4}{*}{ Location* } & \multicolumn{3}{|c|}{ Total Alpha Activity } & \multicolumn{3}{|c|}{ Uranium } & \multicolumn{4}{|c|}{ Plutonium } & \multicolumn{4}{|c|}{ Thorium } \\
\hline & & \multirow{3}{*}{$\begin{array}{l}\text { No. of } \\
\text { Samples }\end{array}$} & \multirow{2}{*}{\multicolumn{2}{|c|}{$\mu \mu \mathrm{c} /$ liter }} & \multirow{3}{*}{$\begin{array}{c}\text { No. of } \\
\text { Samples }\end{array}$} & \multirow{2}{*}{\multicolumn{2}{|c|}{ c/liter }} & \multirow{3}{*}{$\begin{array}{c}\text { No. of } \\
\text { Samples }\end{array}$} & \multicolumn{3}{|c|}{ Samples $>0.2 \mu \mu \mathrm{c} / 1$} & \multirow{3}{*}{$\begin{array}{c}\text { No. of } \\
\text { Samples }\end{array}$} & \multicolumn{3}{|c|}{ Samples $>0.2 \mu \mu \mathrm{c} / 1$} \\
\hline & & & & & & & & & \multirow{2}{*}{ No. } & \multicolumn{2}{|c|}{$\mu \mu \mathrm{c} /$ liter } & & \multirow{2}{*}{ No } & \multicolumn{2}{|c|}{$\mu$ mac/liter } \\
\hline & & & Max. & Av. & & Max. & Av. & & & Max. & Av. & & & Max. & Av. \\
\hline January & A & 1 & 6.4 & -- & 0 & $\cdots$ & - & 1 & 0 & -. & - & 1 & 0 & -. & $\ldots$ \\
\hline January & B & 12 & 25.8 & 10.2 & 2 & 14.0 & 13.7 & 2 & 2 & 8.8 & 5.2 & 2 & 2 & 0.6 & 0.5 \\
\hline February & A & 4 & 8.3 & 4.1 & 4 & 6.8 & 2.9 & 1 & 0 & -- & -- & 1 & 0 & $\ldots$ & -. \\
\hline February & B & 12 & 19.2 & 8.9 & 4 & 22.0 & 124 & 2 & 2 & 6.6 & 5.1 & 2 & 1 & 04 & $\rightarrow$ \\
\hline March & A & 4 & 2.3 & 1.8 & 4 & 1.4 & 1.1 & 2 & 0 & -- & -- & 2 & 0 & $\cdots$ & -- \\
\hline March & B & 13 & 145 & 21 & 5 & 151 & 50 & 2 & 2 & 1.7 & 1.2 & 2 & 2 & 2.6 & 1.6 \\
\hline April & A & 4 & 3.8 & 2.3 & 4 & 1.8 & 1.4 & 2 & 0 & -. & -- & 2 & 0 & -- & $\ldots$ \\
\hline April & B & 13 & 246 & 29 & 4 & 240 & 78 & 2 & 2 & 5.3 & 2.8 & 2 & 1 & 0.6 & - \\
\hline May & A & 5 & 2.0 & 1.7 & 5 & 1.5 & 1.2 & 2 & 0 & - & -- & 2 & 0 & -- & -- \\
\hline May & B & 14 & 32.1 & 9.4 & 4 & 7.0 & 5.8 & 2 & 1 & 0.2 & $-\infty$ & 2 & 1 & 0.4 & $\ldots$ \\
\hline June & A & 4 & 3.2 & 3.0 & 4 & 2.1 & 1.8 & 2 & 0 & -. & - & 2 & 0 & -. & $\infty$ \\
\hline June & B & 12 & 10.5 & 6.0 & 4 & 6.5 & 4.7 & 2 & 1 & 0.5 & -. & 2 & 0 & -. & -- \\
\hline July & A & 5 & 5.3 & 2.6 & 5 & 3.4 & 1.4 & 2 & 0 & - & - & 2 & 1 & 0.3 & -. \\
\hline July & B & 13 & 39.4 & 9.6 & 5 & 37.2 & 12.4 & 2 & 0 & -- & - & 2 & 0 & - & - \\
\hline August & A. & 4 & 2.1 & 1.4 & 4 & 1.3 & 0.9 & 2 & 0 & - & $-\cdots$ & 2 & 1 & 0.2 & - \\
\hline August & $B$ & 13 & 82.0 & 10.6 & 4 & 42.0 & 31.4 & 2 & 1 & 0.3 & $\cdots$ & 2 & 1 & 0.2 & -- \\
\hline September & A & 4 & 6.6 & 3.4 & 4 & 4.1 & 1.8 & 2 & 0 & - & - & 2 & 0 & $=-$ & $-\infty$ \\
\hline September & B & 12 & 14.1 & 5.7 & 4 & 6.8 & 5.5 & 2 & 2 & 1.0 & 0.7 & 2 & 1 & $\ldots$ & - \\
\hline October & A & 5 & 11.4 & 5.3 & 5 & 4.6 & 3.1 & 2 & 0 & - & - & 2 & 1 & 1.2 & $-\infty$ \\
\hline October & B & 13 & 25.6 & 9.9 & 5 & 25.0 & 17.1 & 3 & 3 & 0.2 & 0.2 & 3 & 0 & -- & -- \\
\hline November & A & 4 & 4.9 & 2.6 & 4 & 2.9 & 2.4 & 2 & 0 & - & - & 2 & 1 & 0.7 & .. \\
\hline November & B & 11 & 24.1 & 10.8 & 4 & 35.0 & 25.1 & 3 & 3 & 25.0 & 10.0 & 3 & 1 & 0.4 & $\ldots$ \\
\hline December & A & 3 & 3.6 & 2.6 & 3 & 2.9 & 2.4 & 2 & 0 & $-\infty$ & - & 2 & 1 & 1.1 & -- \\
\hline December & B & 12 & 19.1 & 9.2 & 3 & 17.5 & 13.6 & 2 & 2 & 1.0 & 0.7 & 2 & 0 & -- & -- \\
\hline Average & A & & $\begin{array}{r}2 \\
12\end{array}$ & & & $\begin{array}{r}1 \\
23\end{array}$ & & & & 2 & & & & 0 & \\
\hline
\end{tabular}

* Location $\mathrm{A}$ is above the ANL site.

Location $B$ is below the ANL waste-water outfall. 
for unknown alpha activity. As was found during previous years, uranium was the principal contaminant contributed by Argonne waste water. The average uranium concentration, $23 \mu \mu \mathrm{c} / 1$, was only $0.3 \%$ of the MPC, while the maximum concentration found amounted to about $4 \%$ of the MPC. Except for a few samples, uranium analyses were performed on samples collected from both locations on the same day and, therefore, the average results give approximately the uranium contributed by Laboratory waste water on the days of sample collection. The average uranium concentration was higher than the total alpha activity for two reasons. Samples that contained concentrations of total alpha activity above average were usually analyzed for uranium, and the self-absorption corrections used to obtain the total activity from the counting rates are about $15 \%$ low for normal uranium.

Some of the below-outfall samples also contained detectable amounts of plutonium. while none was detected in the above-site samples. It can, therefore, be concluded that the plutonium was also added to the stream in Laboratory waste water. The highest plutonium concentration $(25 \mu \mu \mathrm{c} / 1)$ was only $16 \%$ of the MPC, while the average concentration, $2.3 \mu \mu \mathrm{c} / 1$, was $1.4 \%$ of the MPC. Small amounts of thorium isotopes were found in some of the samples from both locations, but did not contribute greatly to the total activity of the stream. The concentrations ranged from 0.2 to $2.6 \mu \mu \mathrm{c} / 1$, and the isotopic composition of the thorium was not investigated. A few of the below outfall samples probably contained thorium from Argonne waste water.

Monthly summaries of the beta activities in Sawmill Creek water are given in Table XI. Total beta activity below the outfall averaged $45 \mu \mu \mathrm{c} / 1$, or $16 \mu \mu \mathrm{c} / 1$ higher than above site. However, the magnitude and dixection of this difference varied considerably from time to time because of the variable contribution of fallout and ANL waste water to the total beta activity in the stream. The origin of the beta activity found in the stream could usually be determined by comparing 1) samples collected on the same day from both locations, 2) samples collected during periods of high and low fission product activity in air and rainfall, and 3) results of specific fission product and uranium analyses. The average beta activity below the outfall on days when samples from both locations were analyzed was $42 \mu \mu \mathrm{c} / 1$, or $13 \mu \mu \mathrm{c} / 1$ higher than above the site. Thus, the average in crease in the beta activity of the Creek due to Argonne waste water was $13 \mu \mu \mathrm{c} / 1$. An approximately equal contribution was made by fallout activity. From July through October higher concentrations of beta activity were often found above the site. This was due to the dilution of high concentrations of fallout activity in Sawmill Creek by Argonne waste water containing smaller concentrations of beta activity. The results of specific fission product analyses also provide evidence for this dilution effect. Fallout activ ity was also highest in air and rain during this period. However, even during this period, beta activity originating in Argonne waste water could be 
TAPLF XI

NORUOI ATIIF BETA RADIOACTIYITIES IN SAWMIIL CREFL WATH 195

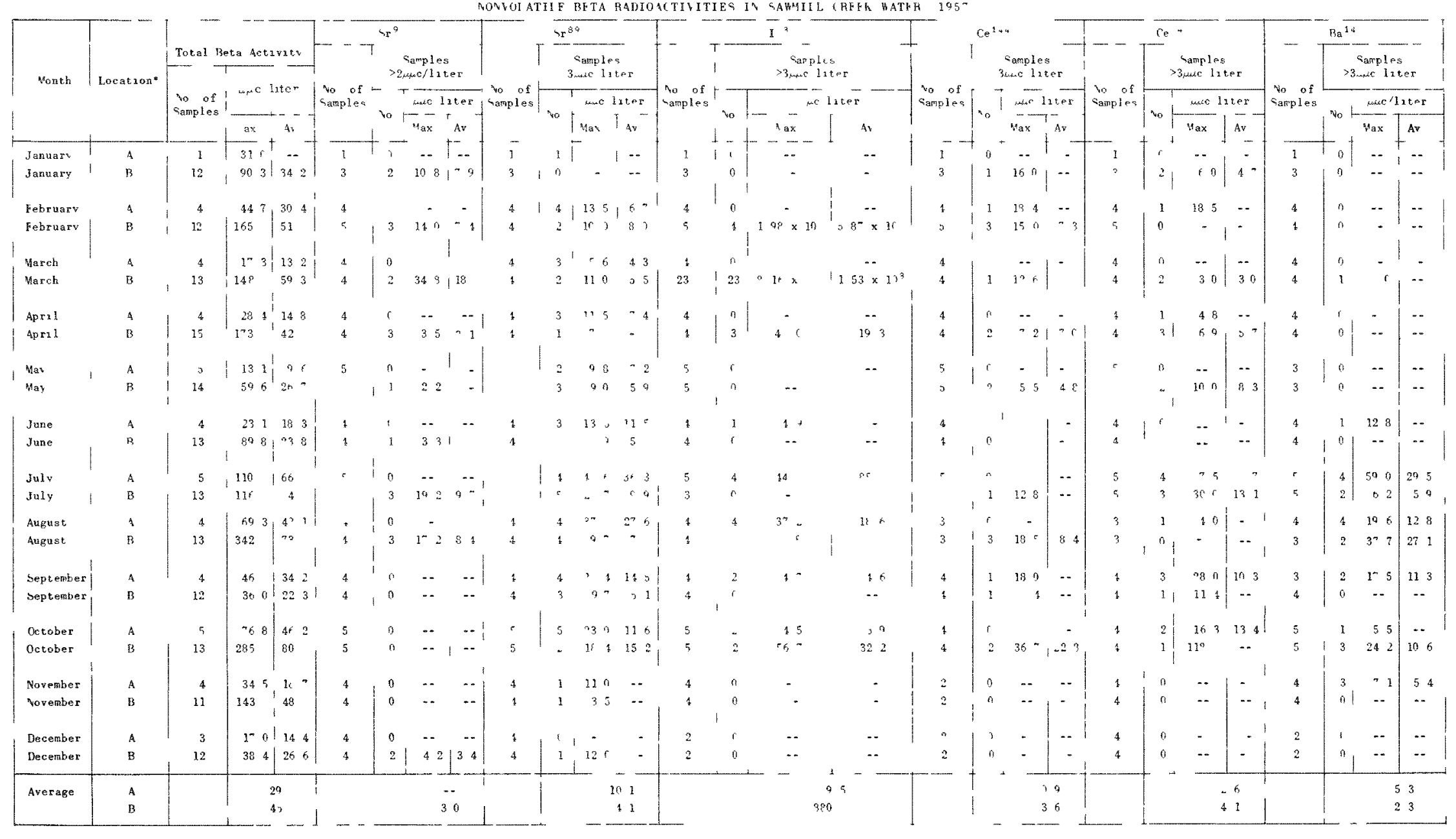

"Location A Is above the Avi oxte
Location B 15 below the ANL, wasto 
detected below the outfall from the results of fission product analyses. $\mathrm{Sr}^{90}$ was found below the outfall during July and August (as well as during other months), but was never detected in concentrations greater than $2 \mu \mu \mathrm{c} / 1$ above site. The $\mathrm{Sr}^{90}$ concentration averaged $3.0 \mu \mu \mathrm{c} / 1$, or about $4 \%$ of the $\mathrm{MPC}^{\circ}$ the maximum $\mathrm{Sr}^{90}$ concentrations found was $34.8 \mu \mu \mathrm{c} / 1$, or about $43 \%$ of the MPC. The longer-lived cerium isotope, $275-d \mathrm{Ce} \mathrm{e}^{144}$ was also found below the outfall on days when it was not detected above site. Except for $\mathrm{Sr}^{90}$, all the fis sion products for which analyses were made were added to the stream at various times by both fallout activity and waste water. All results of fission product analyses were corrected for decay to the time of collection of the sample. As indicated in the table, large concentrations of $8.1-\mathrm{d} \mathrm{I}^{131}$ were found in below-site water during February and March, and this activity was added to the stream in Laboratory waste water. This was confirmed by the presence of iodine activity in waste water before it was added to the stream, and the absence of radioiodine in above-site water. Approximately equal concentrations of $21-\mathrm{h} \mathrm{I}^{133}$ were also found in each sample, indicating that the iodine activities were of recent origin, more recent than found in fallout during the year. This iodine activity originated in a uranium sample undergoing irradiation in the CP-5 reactor and was removed from other fission products by virtue of its volatility. The average $I^{131}$ concentration in below-site water during February and March was equivalent to about $120 \%$ of the MPC: the average for the entire year, $880 \mu \mu \mathrm{c} / 1$, was about $30 \%$ of the MPC. Radioiodine was not observed in the total beta activity, since volatile elements are removed in the preparation of the sample for total beta counting.

Significant amounts of beta activity from the UXI and $\mathrm{UX}_{2}$ daughters of uranium were also found in Creek water below the outfall; this was added in Laboratory waste water. In some samples uranium that had been recently separated from its daughters was found, and the daughter growth was observed. This is additional evidence that below-outfall water contained uranium from Laboratory operations.

Sawmill Creek empties into the Des Plaines River about 500 yards downstream from the waste-water outfall, and the effect of Sawmill Creek water on the radioactivity in the Des Plaines River was determined by sampling the River above and below the mouth of Sawmill Creek at approximately monthly intervals. The results are given in Table XII. The alpha activities and uranium concentrations in water from both locations were low, and no significant or consistent differences between the two locations could be noted. The alpha activities, about $2 \mu \mu \mathrm{c} \alpha / 1$ and about $1.4 \mu \mu \mathrm{cU} / 1$, were similax to those found during previous years. The beta activities were more variable due to fission product fallout. Increases ranging from 20 to $100 \mu \mu \mathrm{c} / 1$ were found during the months of high fallout, July through October. Beta activity due to contamination added to Sawmill Creek could not be detected in any of the 
TABLE XII

NONVOLATILE RADIOACTIVITIES IN DES PLAINES RIVER, 1957

\begin{tabular}{|c|c|c|c|c|}
\hline $\begin{array}{c}\text { Date } \\
\text { Collected }\end{array}$ & Location* & $\begin{array}{c}\text { Alpha Activity } \\
(\mu \mu \mathrm{c} / \text { liter })\end{array}$ & $\begin{array}{l}\text { Uranium } \\
(\mu \mu \mathrm{c} / \text { liter })\end{array}$ & $\begin{array}{c}\text { Beta Activity } \\
(\mu \mu \mathrm{c} / \text { liter })\end{array}$ \\
\hline January 16 & A & 1.0 & 0.7 & 18.3 \\
\hline January 16 & $\mathrm{~B}$ & 1.0 & 1.0 & 20.5 \\
\hline February 20 & A & 3.0 & 0.6 & 25.9 \\
\hline February 20 & B & 1.6 & 1.1 & 19.1 \\
\hline March 20 & A & 1.9 & 0.7 & 29.8 \\
\hline March 20 & B & 1.7 & 0.8 & 25.2 \\
\hline April 17 & $\mathrm{~A}$ & 1.5 & 0.9 & 19.7 \\
\hline April 17 & $\mathrm{~B}$ & 1.3 & 1.2 & 15.9 \\
\hline May 15 & A & 1.6 & 1.4 & 18.5 \\
\hline May 15 & B & 0.7 & 1.2 & 6.7 \\
\hline June 19 & A & 1.7 & 1.1 & 25.0 \\
\hline June 19 & B & 1.1 & 1.4 & 19.4 \\
\hline June 26 & A & 1.7 & - & 18.1 \\
\hline June 27 & $\mathrm{~B}$ & 1. 0 & - & 11.5 \\
\hline July 17 & A & 2.5 & 1.2 & 80.8 \\
\hline July 17 & B & 2.1 & 0.7 & 70.7 \\
\hline August 21 & A & 2.4 & 2.0 & 41.0 \\
\hline August 21 & B & 1.9 & 1.7 & 41.0 \\
\hline September 18 & A & 4.1 & 2.3 & 115 \\
\hline September 18 & $\mathrm{~B}$ & 1.9 & 1.4 & 54.9 \\
\hline October 16 & A & 1.6 & 1.7 & 69.9 \\
\hline October 16 & B & 1.2 & 1.3 & 32.7 \\
\hline November 20 & A & 2.1 & 1.4 & 16.0 \\
\hline November 20 & B & 1.7 & 2.0 & 17.9 \\
\hline December 18 & A & 3.1 & 2.2 & 14.0 \\
\hline December 18 & B & 3.6 & 2.5 & 12.3 \\
\hline \multirow[t]{2}{*}{ Average } & A & 2.2 & 1.4 & 38 \\
\hline & $B$ & 1.6 & 1.4 & 27 \\
\hline
\end{tabular}

* Location $A$ is approximately 5 miles upstream from the mouth of Sawmill Creek.

Location $B$ is approximately 2.5 miles downstream from the mouth of Sawmill Creek. 
samples from the River. There is apparently sufficient dilution so that the radioactivity in Sawmill Creek does not contribute significantly to the alpha or beta activity in the Des Plaines River.

\section{Other Water}

The total activities found in several ponds on the ANL site are summarized in Table XIII. Except for the storage lagoon, the alpha activities were normal, being about $2 \mu \mu \mathrm{c} / 1$, and similar to those found in other years. The beta activities in these samples were also affected by fallout, notably in August. A difference in the origin of the beta activity could be deduced from the decay rate. The beta activities in the natural ponds decayed to $10-15 \mu \mu \mathrm{c} / 1$ in six months regardless of the original activity, and the increases above these values were apparently due to fallout. The beta activities in the storage lagoon samples decayed only $15 \%$ in six months.

TABLE XIII

NONVOLATILE RADIOACTIVITIES

IN PONDS ON ANL SITE, 1957

\begin{tabular}{|c|c|c|c|c|c|}
\hline \multirow{2}{*}{ Month } & \multirow{2}{*}{$\begin{array}{l}\text { No. of } \\
\text { Samples }\end{array}$} & \multicolumn{2}{|c|}{$\begin{array}{c}\text { Alpha Activity } \\
(\mu \mu \mathrm{c} / 1)\end{array}$} & \multicolumn{2}{|c|}{$\begin{array}{c}\text { Beta Activity } \\
(\mu \mu \mathrm{c} / 1)\end{array}$} \\
\hline & & Max. & Av. & Max. & Av. \\
\hline April & 3 & 2.5 & 2.0 & 40.0 & 30.6 \\
\hline June & 4 & 3.01 & $\begin{array}{l}1.4 \\
0.8^{2}\end{array}$ & $37.6^{1}$ & $\begin{array}{l}27.7 \\
24.4^{2}\end{array}$ \\
\hline August & 3 & 1.6 & 0.9 & 74.1 & 54.1 \\
\hline December & 4 & $6.0^{1}$ & $\begin{array}{l}3.0 \\
1.9^{2}\end{array}$ & $60.9^{1}$ & $\begin{array}{l}27.8 \\
16.5^{2}\end{array}$ \\
\hline Average & & \multicolumn{2}{|c|}{$\begin{array}{l}2.0 \\
1.4^{2}\end{array}$} & \multicolumn{2}{|c|}{$\begin{array}{l}34.0 \\
22.4^{2}\end{array}$} \\
\hline
\end{tabular}

${ }^{1}$ Storage lagoon for contaminated waste water.

2 Average excluding results for storage lagoon in June and December.

Under very unusual circumstances there is a possibility that Laboratory waste water from some buildings on the site may enter the drinking or domestic water piping systems within the buildings. 
Because of this, both types of water from many of the buildings were sampled on the same day. Twenty-one samples were analyzed, and all the results were uniformly low. The alpha activities ranged from $0.2 \pm 0.2$ to $0.6 \pm 0.3 \mu \mu \mathrm{c} / 1$ and the beta activities ranged from $2.7 \pm 0.5$ to $6.5 \pm 1.0 \mu \mu \mathrm{c} / 1$. These concentrations are essentially the same as those found in untreated Lake Michigan water.

The total activities in water from lakes and streams near the ANL site are given in Tables XIV and XV. The alpha activities were normal and ranged from 0.2 and $3.9 \mu \mu \mathrm{c} / 1$. As was found previously, the highest alpha activities were generally found in the Du Page River at Naperville and the lowest in Lake Michigan. The average beta activity, about $21 \mu \mu \mathrm{c} / 1$, was similar to that found in most other years. The highest beta activities were found in the October samples as a result of increased fallout. The fallout added an average of about $15 \mu \mu \mathrm{c} / 1$ to the beta activity. An abnormally high concentration of beta activity, $103 \mu \mu \mathrm{c} / 1$, was found in the Du Page River at Channahan in December. The activity decayed rapidly and had decreased to $39 \mu \mu \mathrm{c} / 1$ in two months. All other samples collected near the Laboratory in December contained less than $20 \mu \mu \mathrm{c} / 1$.

There was no correlation between activity and distance from the Laboratory in any of the samples collected near the Laboratory. The Des Plaines River empties into the Illinois River at McKinley Woods, and water was collected at this point, as well as from the Illinois River at Morris and at Starved Rock State Park. No unusual activities were found at any of the se locations.

Water was also collected from the Sanitary and Ship Canal near Lemont in November. On November 5, several curies of $85-\mathrm{d} \mathrm{Sc}{ }^{45}$ and tritium were added to the Canal by a group from Northwestern University in a tracer experiment. The scandium and tritium activities found in the samples on November 5 were $360 \mu \mu \mathrm{c} / 1$ and $3.3 \times 10^{5} \mu \mu \mathrm{c} / 1$, respectively. Neither nuclide could be detected on the following day.

The results obtained for the 100 mile samples are given in Table XVI. The average alpha and beta activities, 0.7 and $16 \mu \mu \mathrm{c} / 1$, respectively, were normal and similar to those found previously. The December samples from Shafer Lake and Pipestone Lake had abnormally high concentrations of beta activity. This was probably due to fallout in those areas, since the samples decayed in three months from 20 to $9 \mu \mu \mathrm{c} / 1$ in the Shafer Lake sample and from 80 to $30 \mu \mu \mathrm{c} / 1$ for the Pipestone Lake sample.

\section{Bottom Silt}

The results of measurements of total activity on bottom silt from Sawmill Creek and the DesPlaines River are given in Table XVII. 
TABLE XIV

NONVOLATILE RADIOACTIVITES IN LAKES AND STREAMS NEAR ANL, 1957

\begin{tabular}{|c|c|c|c|c|c|c|c|c|c|c|c|}
\hline \multirow{2}{*}{ Lake or Stream } & \multirow{2}{*}{ Location } & \multicolumn{2}{|c|}{ April } & \multicolumn{2}{|c|}{ June } & \multicolumn{2}{|c|}{ October } & \multicolumn{2}{|c|}{ December } & \multicolumn{2}{|c|}{ Average } \\
\hline & & $\mu \mu \mathrm{c} a / 1$ & $\mu \mu \mathrm{c} \beta / 1$ & $\mu \mu \mathrm{c} a / 1$ & $\mu \mu \mathrm{c} \beta / 1$ & $\mu \mu \mathrm{c} \alpha / 1$ & $\mu \mu c \beta / 1$ & $\mu \mu c \alpha / 1$ & $\mu \mu \subset \beta / 1$ & $\mu \mu \mathrm{ca} / 1$ & $\mu \mu c \beta / 1$ \\
\hline Desplaines River ${ }^{1}$ & Irving Park Road & 2.0 & 11.5 & 3.7 & 30.8 & 1.6 & 21.2 & 1.9 & 18.6 & 2.3 & 20.5 \\
\hline DesPlaines River ${ }^{1}$ & Willow Springs & 1.5 & 19.7 & 1.7 & 18.1 & 1.6 & 69.9 & 3.1 & 14.0 & 2.0 & 30.4 \\
\hline Desplaines River ${ }^{2}$ & Lemont & 1.3 & 15.9 & 1.0 & 11.5 & 1.2 & 32.7 & 3.6 & 12.3 & 1.8 & 18.1 \\
\hline Desplaines River ${ }^{2}$ & Romeoville & 1.8 & 11.0 & 1.4 & 11.1 & 2.5 & 40.8 & 2.2 & 13.8 & 2.0 & 19.2 \\
\hline DesPlaines River ${ }^{2}$ & Brandon Road, Joliet & - & -- & 0.5 & 9.7 & $\infty$ & - & $\ldots$ & - & 0.5 & 9.7 \\
\hline DuPage River & Naperville & 3.9 & 17.2 & 2.7 & 24.3 & 2.2 & 20.1 & 3.5 & 14.2 & 3.1 & 19.0 \\
\hline DuPage River & Channahon & 2.2 & 11.4 & 2.9 & 10.8 & 1.5 & 78.3 & 2.6 & 103 & 2.3 & 51 \\
\hline IIl. \& Mich. Canal & Channahon & 1.3 & 22.5 & 1.3 & 11.8 & - & - & -- & $-\infty$ & 1.3 & 17.2 \\
\hline Fox River & Aurora & $\cdots$ & $\infty$ & 0.5 & 10.5 & $\infty$ & $\ldots$ & 1.3 & 15.1 & 0.9 & 12.8 \\
\hline Flag Creek & Willow Springs & 0.7 & 13.2 & 1.2 & 32.5 & - & -- & -- & -- & 1.0 & 22.9 \\
\hline Saganaskee Slough & 104th Ave. \& Sag Canal & 1.0 & 27.8 & - & -- & 0.5 & 23.6 & 0.5 & 18.8 & 0.7 & 23.4 \\
\hline Cal-Sag Canal & U.S. Rt. $83 \& 86$ th Ave. & 0.6 & 13.2 & -- & $\infty$ & 0.5 & 13.3 & - & - & 0.6 & 13.3 \\
\hline Long Run Creek & 135th St. \& Archer Ave. & 1.0 & 8.0 & -- & -- & 1.0 & 7.9 & -- & $-\infty$ & 1.0 & 8.0 \\
\hline Sauk Trail Lake & 226th St. \& Western Ave. & 1.2 & 10.3 & - & $\cdots$ & 1.0 & 20.4 & $-\infty$ & -- & 1.1 & 15.4 \\
\hline $\begin{array}{l}\text { Confluence - DesPlaines, } \\
\text { II., \& Kankakee Rivers }\end{array}$ & McKinley Woods & 1.4 & 9.9 & 2.2 & 16.3 & 0.8 & 8.9 & 1.4 & 10.7 & 1.5 & 11.5 \\
\hline Illinois River & Morris & -- & - & 1.0 & 8.1 & -- & $-\infty$ & 1.2 & 7.7 & 1.1 & 7.9 \\
\hline San. Ship Canal & Lemont & $-\infty$ & -- & -- & - & 2.1 & 35.0 & - & $\ldots$ & 2.1 & 35.0 \\
\hline Calumet Lake & 111 th St., Chicago & $\cdots$ & -. & -- & -. & 0.7 & 25.1 & 1.1 & 17.2 & 0.9 & 21.2 \\
\hline Lake Michigan & 98th St., Chicago & $-\infty$ & $-\infty$ & $-\infty$ & -- & 0.2 & 4.3 & 0.4 & 6.4 & 0.3 & 5.4 \\
\hline McGinnis Slough & U.S. Rt. $45 \&$ IIl. Rt. 7 & - & $=$ & $-\infty$ & - & 1.1 & 25.8 & $\ldots$ & $\ldots$ & 1.1 & 25.8 \\
\hline
\end{tabular}

1 Above mouth of Sawmill Creek

${ }^{2}$ Below mouth of Sawmill Creek 
TABLE XV

NONVOLATILE RADIOACTIVITIES IN LAKES

AND STREAMS NEAR ANL, 1957

\begin{tabular}{|c|c|c|c|c|c|c|}
\hline \multirow[t]{2}{*}{$\begin{array}{c}\text { Distance From } \\
\text { ANL (miles) }\end{array}$} & \multirow[t]{2}{*}{ Date } & \multirow[t]{2}{*}{$\begin{array}{l}\text { No. of } \\
\text { Samples }\end{array}$} & \multicolumn{2}{|c|}{$\begin{array}{c}\text { Alpha } \\
\text { Activity } \\
\mu \mu c / \text { liter }\end{array}$} & \multicolumn{2}{|c|}{$\begin{array}{c}\text { Beta } \\
\text { Activity } \\
\mu \mu c / \text { liter }\end{array}$} \\
\hline & & & Max. & Av. & Max. & Av. \\
\hline 10 & April & 7 & 1.8 & 1.1 & 27.8 & 15.5 \\
\hline 10 & June & 4 & 1.7 & 1.3 & 32.5 & 18.3 \\
\hline 10 & October & 8 & 2.5 & 1.3 & 69.9 & 31.1 \\
\hline 10 & December & 4 & 3.6 & 2.3 & 18.8 & 14.7 \\
\hline 10 (average) & & & \multicolumn{2}{|c|}{1.4} & \multicolumn{2}{|c|}{21} \\
\hline 25 & April & 5 & 3.9 & 2.1 & 17.2 & 12.1 \\
\hline 25 & June & 8 & 3.7 & 1.8 & 30.8 & 15.3 \\
\hline 25 & October & 7 & 2.2 & 1.1 & 78.3 & 25.5 \\
\hline 25 & December & 8 & 3.5 & 1.7 & 103 & 24.1 \\
\hline 25 (average) & & & \multicolumn{2}{|c|}{1.7} & \multicolumn{2}{|c|}{20} \\
\hline $\begin{array}{l}\text { Annual } \\
\text { Average }\end{array}$ & & & \multicolumn{2}{|c|}{1.5} & \multicolumn{2}{|c|}{21} \\
\hline
\end{tabular}

TABLE XVI

NONVOLATILE RADIOACTIVITIES IN WATER

FROM REFER ENCE SITES, 1957

\begin{tabular}{|l|r|r|r|r|}
\hline \multirow{2}{*}{ Location } & \multicolumn{2}{c|}{ June } & \multicolumn{2}{c|}{ December } \\
\cline { 2 - 5 } & $\mu \mu \mathrm{c} \alpha / 1$ & $\mu \mu \mathrm{c} \beta / 1$ & $\mu \mu \mathrm{c} \alpha / 1$ & $\mu \mu \mathrm{c} / 1$ \\
\hline Lake Geneva, Wisconsin & 0.1 & 5.6 & 0.2 & 4.1 \\
Lake Delavan, Wisconsin & 1.0 & 15.6 & 0.7 & 12.2 \\
Ilinois River, Starved Rock State Park & 0.8 & 10.2 & 1.3 & 15.1 \\
Shafer Lake, Monticello, Indiana & 0.8 & 6.7 & 1.5 & 28.7 \\
Lake Michigan, St. Joseph, Michigan & 0.1 & 3.7 & 0.4 & 4.6 \\
Pipestone Lake, Michigan & 0.1 & 2.6 & 1.1 & 80.3 \\
\hline Average & 0.5 & 7.7 & 0.9 & 24.2 \\
\hline
\end{tabular}


TABLE XVII

NONVOLATILE RADIOACTIVITIES IN BOT TOM SILT FROM

SAWMILL CREEK AND DES PLAINES RIVER, 1957

\begin{tabular}{|c|c|c|c|c|c|c|}
\hline \multirow{2}{*}{$\begin{array}{c}\text { Date } \\
\text { Collected }\end{array}$} & \multicolumn{3}{|c|}{ Sawmill Creek } & \multicolumn{3}{|c|}{ Des Plaines River } \\
\hline & Location 1 & $\mu \mu \mathrm{c} \alpha / \mathrm{g}$ & $\mu \mu \mathrm{c} \beta / \mathrm{g}$ & Location ${ }^{2}$ & $\mu \mu c \alpha / g$ & $\mu \mu c \beta / g$ \\
\hline January 16 & A & - & - & A & 16 & 38 \\
\hline January 16 & $\mathrm{~B}$ & 28 & 48 & $\mathbf{B}$ & 20 & 59 \\
\hline February 20 & A & 20 & 55 & A & 22 & 54 \\
\hline Februaxy 20 & $\mathrm{~B}$ & 28 & 58 & $\mathbf{B}$ & 25 & 55 \\
\hline March 20 & A & 26 & 51 & $\mathbb{A}$ & 21 & 61 \\
\hline March 20 & B & 24 & 48 & $\mathrm{~B}$ & 21 & 46 \\
\hline April 17 & A & 22 & 39 & $\mathbb{A}$ & 20 & 58 \\
\hline April 17 & $\mathrm{~B}$ & 22 & 37 & $\mathrm{~B}$ & 22 & 38 \\
\hline June 19 & A & 23 & 65 & A & 20 & 52 \\
\hline June 19 & $\mathrm{~B}$ & 46 & 67 & $\mathrm{~B}$ & 29 & 61 \\
\hline June 26 & A & 23 & 65 & A & 22 & 69 \\
\hline June 26 & B & 24 & 57 & $\mathrm{~B}$ & 21 & 79 \\
\hline July 17 & $\mathbf{A}$ & 26 & 78 & A & 25 & 155 \\
\hline July 17 & $\mathrm{~B}$ & 11 & 35 & B & 22 & 49 \\
\hline August 21 & A & 18 & 48 & A & 22 & 56 \\
\hline August 21 & B & 61 & 110 & B & 21 & 73 \\
\hline October 2 & $\mathbb{A}$ & 23 & 58 & A & 22 & 85 \\
\hline October 2 & B & 126 & 108 & $\mathrm{~B}$ & 22 & 96 \\
\hline October 16 & A & 26 & 67 & $A$ & - & - \\
\hline October 16 & B & 374 & 364 & $\mathrm{~B}$ & 25 & 60 \\
\hline December 18 & A & 25 & 75 & A & 17 & 57 \\
\hline December 18 & $B$ & 398 & 1157 & B & 23 & 87 \\
\hline \multirow[t]{2}{*}{ Average } & A & 23 & 60 & A & 21 & 69 \\
\hline & B & 104 & 190 & B & 23 & 64 \\
\hline
\end{tabular}

${ }^{1}$ Location $A$ is above the ANL site.

Location $B$ is below the ANL waste-water outfall.

${ }^{2}$ Location $A$ is approximately 5 miles above the mouth of Sawmill Creek. Location $B$ is approximately 2.5 miles below the mouth of Sawmill Creek. 
Normal values for bottom silt from these two streams range from about 10 to $30 \mu \mu \mathrm{c} \alpha / \mathrm{g}$ and 50 to $90 \mu \mu \mathrm{c} / \mathrm{g}$, depending on the nature of the individual sample. Samples from Sawmill Creek above the site throughout the year and from below the outfall from January through July were normal. In creases ranging from 30 to $300 \mu \mu \mathrm{c} \alpha / \mathrm{g}$ and 20 to $1,000 \mu \mu \mathrm{c} \beta / \mathrm{g}$ were found in the below-site samples collected from August through December. The increased activities in these below-outfall samples were derived primarily from radioactivity added to the stream in Argonne waste water, although some of the beta activity may have been derived from fallout. This is confirmed by the results given in Table XVIII. Abnormally high concentrations of uranium, plutonium, and strontium-90 were found only in the samples collected below the outfall, and these nuclides were also present in abnormally high concentrations in below-outfall water. The plutonium/uranium ratio in the below-site bottom silt was about 15 times greater than has generally been found in the water from that location. Since the relative concentrations of a particular nuclide in water and bottom silt will depend on many factors, including the chemistry of the element, the nature of the stream bed, and the chemical composition of the water, the concentration in bottom silt can be predicted from the water concentration only if all the variables are correctly evaluated. However, for long-lived nuclides the bottom silt should be a sensitive qualitative index of the activity added to the Creek in waste water over a period of years.

TABLE XVIII

RADIOACTIVITIES IN BOTTOM SILT, 1957

\begin{tabular}{|c|c|c|c|c|c|c|c|}
\hline Location & $\begin{array}{c}\text { Date } \\
\text { Collected }\end{array}$ & $\begin{array}{l}\text { Alpha } \\
\text { Activity, } \\
\mu \mu c / g\end{array}$ & $\begin{array}{c}\text { Beta } \\
\text { Activity, } \\
\mu \mu \mathrm{c} / \mathrm{g}\end{array}$ & $\begin{array}{l}\text { Uranium, } \\
\mu \mathrm{g} / \mathrm{g}\end{array}$ & $\begin{array}{l}\text { Plutonium, } \\
\mu \mu \mathrm{c} / \mathrm{g}\end{array}$ & $\begin{array}{c}\text { Thorium, } \\
\mu \mu \mathrm{c} / \mathrm{g}\end{array}$ & $\begin{array}{c}S r_{2}^{90} \\
\mu \mu_{c} / g\end{array}$ \\
\hline Sawmill Creek - below outfall & $10-16$ & 374 & 364 & 85 & 93 & 7 & 8.4 \\
\hline Sawmill Creek - below outfall & $12-18$ & 398 & 1157 & 101 & 149 & 9 & 13.5 \\
\hline Sawmill Craek - above site & $12-18$ & 25 & 75 & 2.8 & - & - & $<1.5$ \\
\hline Pond on ANL site & $4-27$ & 29 & 116 & 2.4 & $<0.3$ & 3 & $<1.5$ \\
\hline Des Plaines River - above site & $6-26$ & 25 & 147 & 2.7 & - & - & $<1.5$ \\
\hline Des Plaines River - below site & $9-24$ & 13 & 68 & 2.3 & - & - & $<1.5$ \\
\hline Des Plaines River - below site & $9-25$ & 28 & 198 & 2.3 & - & - & $<1.5$ \\
\hline Des Plaines River - above site & $9-25$ & 24 & 142 & 2.5 & - & - & $<1.5$ \\
\hline Du Page River, Naperville & $12-17$ & 85 & 75 & 4.4 & - & - & $<1.5$ \\
\hline
\end{tabular}

The relatively high concentration of alpha activity $(85 \mu \mu \mathrm{c} / \mathrm{g})$ in the sample from the DuPage River is reflected in the uranium concentration. This sample contained $4.4 \mu \mathrm{gU} / \mathrm{g}$, while all the other samples analyzed (except from below the outfall) contained from 2.3 to $2.8 \mu \mathrm{gU} / \mathrm{g}$. Similar results have been obtained from the Du Page River in previous years. Above-average concentrations of other naturally occur ring radioactive nuclides have also been found at this location.

Bottom silt from the Des Plaines River averaged essentially the same above and below the ANL site. The averages, about $22 \mu \mu \mathrm{c} \alpha / \mathrm{g}$ and $67 \mu \mu \mathrm{c} \beta / \mathrm{g}$, respectively, were in the range normally found in this 
stream. Thus, the contamination added to Sawmill Creek by ANL waste water did not contribute significantly to the radioactivity in Des Plaines River bottom silt. The concentrations of beta emitters increased during the summer when fallout activity increased. The large diffexences of beta activities in the July samples were probably due to the fact that the above-site samples consisted primarily of clay, while the below-site sample contained a large fraction of sand. It has been generally observed that stream beds consisting of clay contain the largest concentrations of radioactivity, while sand beds contain little activity.

The results of extensive sampling of the beds of Sawmill Creek and the Des Plaines River in September and November are plotted in Figures 11 and 12. The results are consistent with those obtained for the monthly samples. In Sawmill Creek increases in both alpha and beta activities by factors of about three and six, respectively, were found 20 , 25 , and 200 yards downstream from the waste-water outfall. The Creek bed was not uniformly contaminated, and the amount of contamination was not a function of the distance downstream from the outfall. Large variations in activity with time and location were also observed in bottom silt from all streams, probably as a result of variations in flow rate and in the chemical and physical nature of the stream bed. A study of some of the variables is planned.

The survey of the bed of the Des Plaines River did not show any activity that could be attributed to ANL waste water. It should be noted that increases due to ANL waste water should be observed in both the alpha and beta concentrations, as was found in Sawmill Creek bottom silt. The alpha activities ( 9 to $28 \mu \mu \mathrm{c} / \mathrm{g}$ ) were in the normal range. The beta activities showed greater variation $(25$ to $198 \mu \mu \mathrm{c} / \mathrm{g}$ ), presumably due to the varying amounts of fallout. The total beta activities decayed from 20 to $60 \%$ in four months. No pronounced trend in activity with distance could be ascertained, although the average activity was slightly higher from 4,200 to 5,500 yards than farther upstream. The physical characteristics of the bed were variable, and, in general, samples consisting largely of clay contained more activity than samples containing a large percentage of sand.

Bottom silts from ponds on the ANL site were normal, except for samples obtained from the storage lagoon for contaminated waste water. The results are summarized in Table XIX. The bottom silts from the natural ponds contained an average of $26 \mu \mu \mathrm{c} \alpha / \mathrm{g}$ and $66 \mu \mu \mathrm{c} \beta / \mathrm{g}$. The activities in samples from the storage lagoon were 10 to 30 times greater. The bed of the storage lagoon contains a variety of nuclides adsorbed from the contaminated water stored in the lagoon periodically.

The results obtained for bottom silts collected from lakes and streams near the Laboratory are given in Tables XX and XXI. The range of activities and the average activity were similar to those found in 
Figure 11

NONVOLATILE RADIOACTIVITIES IN SAW MILL CREEK BOTTOM SLLT

November, 1957

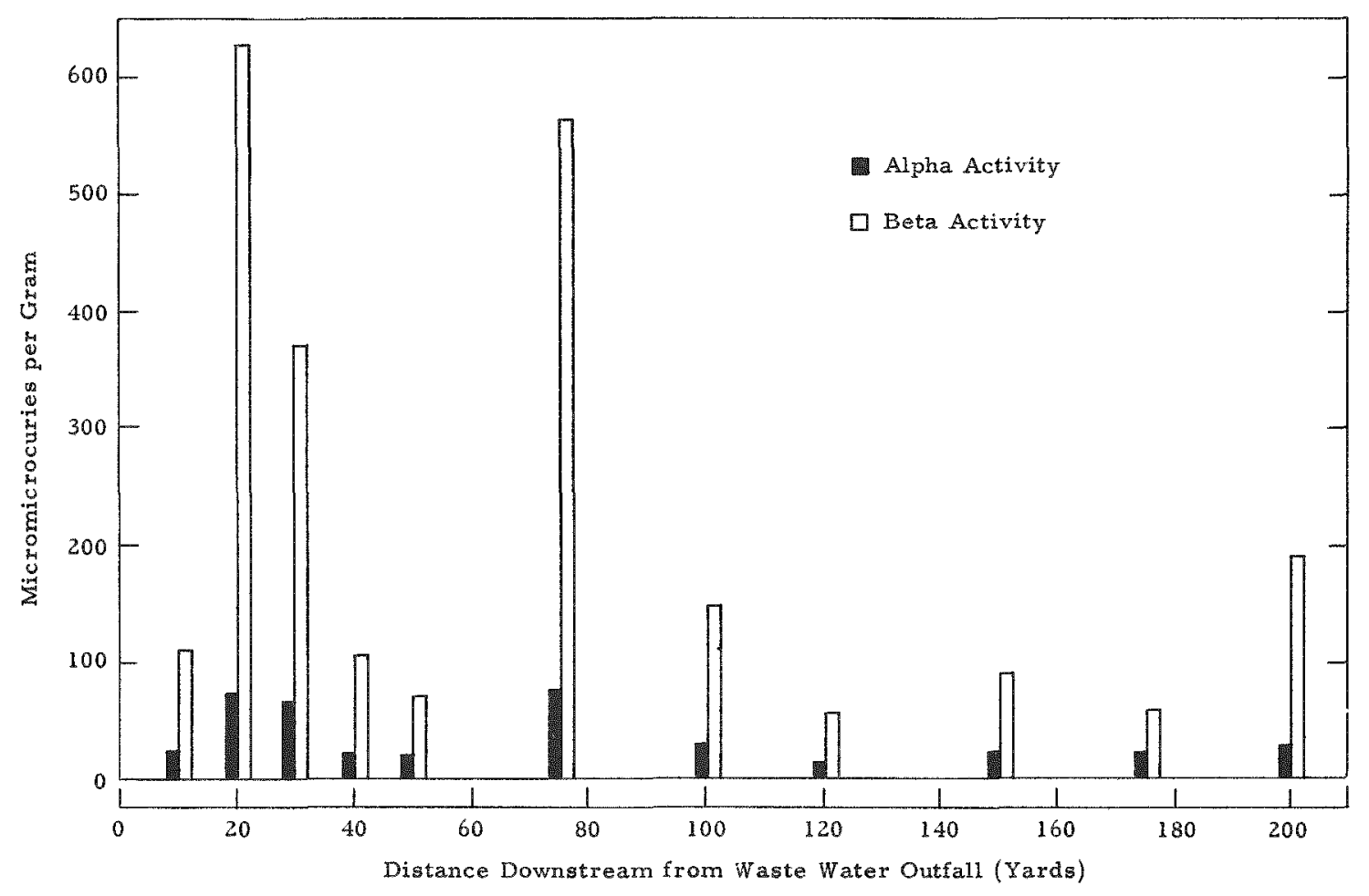

Figure 12

NONVOLATILE RADIOACTIVITIES IN DESPLAINES RIVER BOTTOM SILT

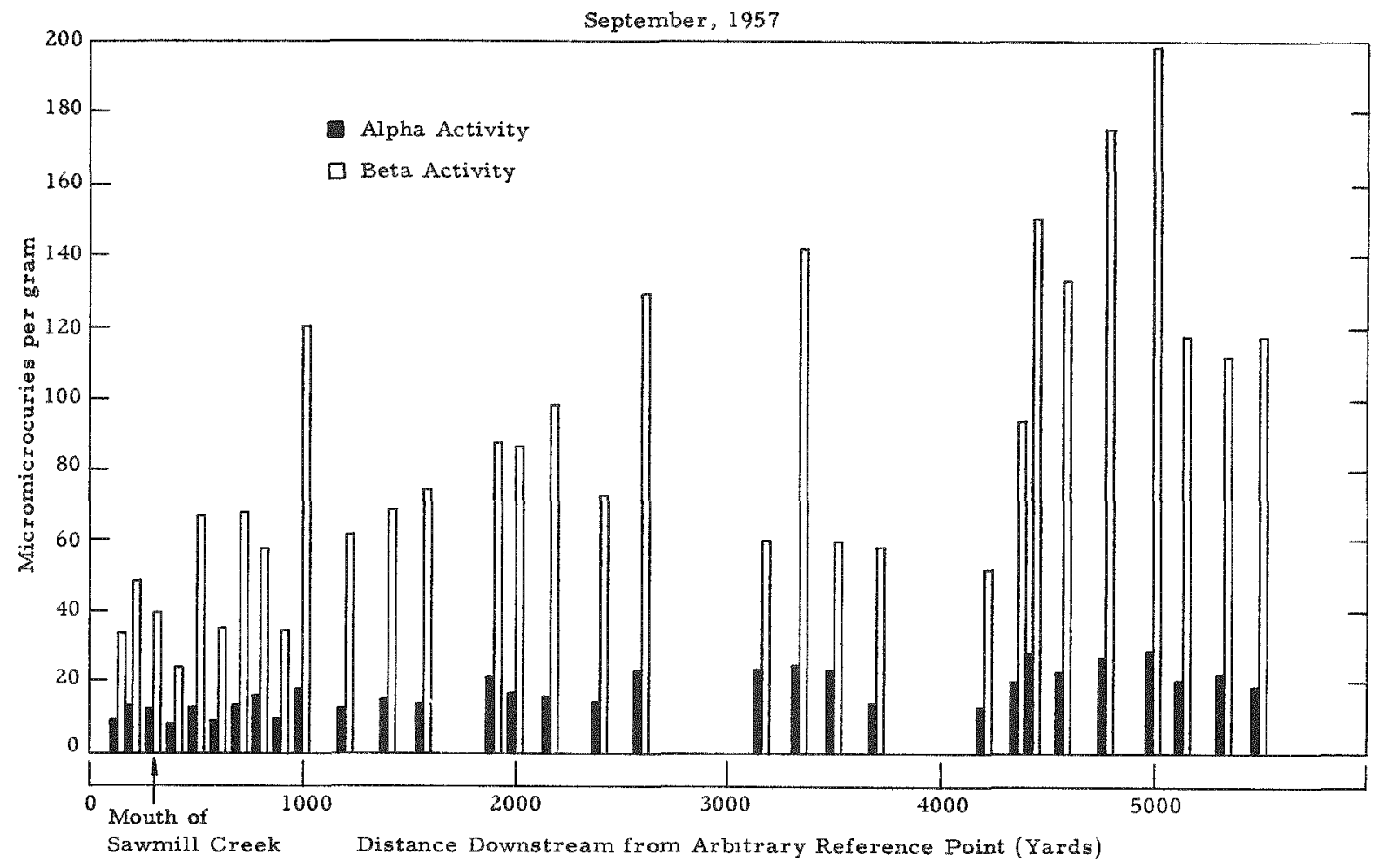


TABLE XIX

NONVOLATILE RADIOACTIVITIES IN BOTTOM

SILT FROM PONDS ON ANL SITE, 1957

\begin{tabular}{|c|c|c|c|c|c|}
\hline \multirow{2}{*}{$\begin{array}{c}\text { Month } \\
\text { Collected }\end{array}$} & \multirow{2}{*}{$\begin{array}{l}\text { No. of } \\
\text { Samples }\end{array}$} & \multicolumn{2}{|c|}{$\begin{array}{c}\text { Alpha } \\
\text { Activity, } \\
\mu \mu \mathrm{c} / \mathrm{g}\end{array}$} & \multicolumn{2}{|c|}{$\begin{array}{c}\text { Beta } \\
\text { Activity, } \\
\mu \mu \mathrm{c} / \mathrm{g}\end{array}$} \\
\hline & & Max. & Av. & Max. & Av. \\
\hline April & 3 & 29 & 28 & 116 & 81 \\
\hline June & 4 & 8451 & $\begin{array}{l}235 \\
272\end{array}$ & 13351 & $\begin{array}{l}382 \\
652\end{array}$ \\
\hline August & 3 & 28 & 26 & 61 & 55 \\
\hline December & 3 & 3611 & $\begin{array}{l}162 \\
23(2)\end{array}$ & $770(1)$ & $\begin{array}{l}298 \\
63(2)\end{array}$ \\
\hline \multicolumn{2}{|l|}{ Average } & \multicolumn{2}{|c|}{$\begin{array}{r}122 \\
262\end{array}$} & \multicolumn{2}{|c|}{$\begin{array}{r}218 \\
662\end{array}$} \\
\hline
\end{tabular}

IStorage lagoon for radioactive waste water.

2Average excluding storage lagoon samples.

previous years. The beta activities in most samples exhibited decay, and the rate of decay was greater for the higher samples. This short-lived activity was presumably due to fallout. The alpha and beta activities in bottom silts from the reference sites (Table XXII) were uniformly low, ranging from 1.5 to $16 \mu \mu \mathrm{c} \alpha / \mathrm{g}$ and 8 to $37 \mu \mu \mathrm{c} \beta / \mathrm{g}$. No abnormal results were obtained. The average values $(6 \mu \mu \mathrm{c} \alpha / \mathrm{g}$ and $21 \mu \mu \mathrm{c} \beta / \mathrm{g})$ were lower than for samples collected near or on the Laboratory site, because the reference site samples were usually sandy and contained very little fission products of recent origin.

E. Soil

The alpha and beta activities found in surface soil on the ANL site during 1957 are summarized in Table XXIII. Most of the samples were in the normal range of $20-30 \mu \mu \mathrm{c} \alpha / \mathrm{g}$ and $40-80 \mu \mu \mathrm{c} \beta / \mathrm{g}$. A few of the samples collected in June, August, and October contained 25 to $150 \mu \mu \mathrm{c} / \mathrm{g}$ more beta activity than normal. These increases, observed only during periods of high fallout, were evidently due to fallout activity. The beta activities in these samples decayed to normal values in about four months, regardless of the original activity, while the alpha activity remained constant. Similar results were observed in samples collected near the Laboratory site during the same periods. One sample collected near a uranium storage shed on the site in December contained $39 \mu \mu \mathrm{c} \alpha / \mathrm{g}$ and $106 \mu \mu \mathrm{c} \beta / \mathrm{g}$, 
TABLE XX

NONVOLATILE RADIOACTIVITIES IN BOTTOM SILTS FROM LAKES AND STREAMS NEAR ANL, 1957

\begin{tabular}{|c|c|c|c|c|c|c|c|c|c|c|c|}
\hline \multirow{2}{*}{ Lake or Stream } & \multirow{2}{*}{ Location } & \multicolumn{2}{|c|}{ April } & \multicolumn{2}{|c|}{ June } & \multicolumn{2}{|c|}{ October } & \multicolumn{2}{|c|}{ December } & \multicolumn{2}{|c|}{ Average } \\
\hline & & $\mu_{\mu} \subset \alpha / 1$ & $\mu \mu \mathrm{c} \rho / 1$ & $\mu \mu c_{a} / 1$ & $\mu \mu \mathrm{c} \beta / 1$ & $\mu \mu c \alpha / 1$ & $\mu \mu \mathrm{c} \beta / 1$ & $\mu \mu \mathrm{ca} / 1$ & $\mu \mu \mathrm{c} \beta / 1$ & $\mu \mu c \alpha / 1$ & $\mu \mu c \beta / 1$ \\
\hline DesPlaines River 1 & Irving Park Road & 31 & 86 & 25 & 147 & 24 & 143 & 22 & 57 & 26 & 108 \\
\hline DesPlaines River 1 & Willow Springs & 16 & 38 & 20 & 52 & - & - & 17 & 57 & 18 & 49 \\
\hline DesPlaines River ${ }^{2}$ & Lemont & 20 & 59 & 29 & 61 & 25 & 60 & 23 & 87 & 24 & 67 \\
\hline DesPlaines River 2 & Romeoville & 29 & 99 & 23 & 73 & 17 & 83 & 24 & 117 & 23 & 93 \\
\hline DesPlaines River ${ }^{2}$ & Brandon Rd., Joliet & - & - & 15 & 37 & - & - & - & - & 15 & 37 \\
\hline DuPage River & Naperville & 41 & 57 & 12 & 21 & 20 & 33 & 84 & 75 & 39 & 47 \\
\hline DuPage River & Channahon & 30 & 64 & 35 & 66 & 16 & 54 & 9 & 23 & 23 & 52 \\
\hline IIl. \& Mich. Canal & Channahon & 29 & 50 & 20 & 35 & - & - & - & - & 25 & 43 \\
\hline Fox River & Aurora & - & - & 21 & 82 & - & - & 3 & 23 & 12 & 53 \\
\hline Flag Creek & Willow Springs & 27 & 79 & 9 & 25 & - & - & - & - & 18 & 52 \\
\hline Saganaskee Slough & 104th Ave. \& Sag Canal & 17 & 49 & - & - & 16 & 65 & - & - & 17 & 57 \\
\hline Long Run Creek & 135th St. \& Archer Ave. & 29 & 72 & - & - & 21 & 91 & - & - & 25 & 82 \\
\hline Sauk Trail Lake & 226th St. \& Western Ave. & 21 & 52 & - & - & 20 & 53 & - & - & 21 & 53 \\
\hline Dlinois River & Morris & - & - & 12 & 35 & - & - & 8 & 24 & 10 & 30 \\
\hline Calumet Lake & 111th St., Chicago & - & - & - & - & 20 & 110 & 21 & 41 & 21 & 76 \\
\hline Lake Michigan & 98th St., Chicago & - & - & - & - & 3 & 26 & - & - & 3 & 26 \\
\hline McGinnis Slough & U.S. Rt. 45 \& Ill. Rt. 7 & - & - & - & - & 27 & 89 & - & - & 27 & 89 \\
\hline
\end{tabular}

1 Above mouth of Sawmill Creek

${ }^{2}$ Below mouth of Sawmill Creek 
TABLE XXI

NONVOLATILE RADIOACTIVITIES IN BOTTOM

SILTS NEAR ANL, 1957

\begin{tabular}{|c|c|c|c|c|c|c|}
\hline \multirow[t]{2}{*}{$\begin{array}{l}\text { Distance From } \\
\text { ANL (miles) }\end{array}$} & \multirow[t]{2}{*}{ Month } & \multirow[t]{2}{*}{$\begin{array}{l}\text { No. of } \\
\text { Samples }\end{array}$} & \multicolumn{2}{|c|}{$\begin{array}{c}\text { Alpha } \\
\text { Activity, } \\
\mu \mu \mathrm{c} / \mathrm{gram}\end{array}$} & \multicolumn{2}{|c|}{$\begin{array}{c}\text { Beta } \\
\text { Activity, } \\
\mu \mu \mathrm{c} / \mathrm{gram}\end{array}$} \\
\hline & & & Max. & Av. & Max. & $A v$. \\
\hline 10 & April & 7 & 41 & 24 & 99 & 62 \\
\hline 10 & June & 5 & 29 & 23 & 73 & 46 \\
\hline 10 & October & 6 & 27 & 21 & 91 & 70 \\
\hline 10 & December & 4 & 84 & 37 & 117 & 84 \\
\hline 10 (average) & & & \multicolumn{2}{|c|}{25} & \multicolumn{2}{|c|}{65} \\
\hline 25 & April & 4 & 31 & 30 & 86 & 68 \\
\hline 25 & June & 6 & 35 & 21 & 147 & 67 \\
\hline 25 & October & 5 & 24 & 17 & 143 & 77 \\
\hline 25 & December & 5 & 22 & 13 & 57 & 34 \\
\hline 25 (average) & & & \multicolumn{2}{|c|}{20} & \multicolumn{2}{|c|}{61} \\
\hline $\begin{array}{l}\text { Annual } \\
\text { Average }\end{array}$ & & & \multicolumn{2}{|c|}{23} & \multicolumn{2}{|c|}{63} \\
\hline
\end{tabular}

TABLE XXII

NONVOLATILE RADIOACTIVITIES IN BOTTOM SILTS FROM REFERENCE SITES, 1957

\begin{tabular}{|c|c|c|c|c|}
\hline \multirow{2}{*}{ Location } & \multicolumn{2}{|c|}{ June } & \multicolumn{2}{|c|}{ December } \\
\hline & $\mu \mu \mathrm{c} \alpha / \mathrm{g}$ & $\mu \mu \mathrm{c} \beta / \mathrm{g}$ & $\mu \mu c \alpha / g$ & $\mu \mu \mathrm{c} \beta / \mathrm{g}$ \\
\hline $\begin{array}{l}\text { Lake Geneva, } \\
\text { Wisconsin }\end{array}$ & 3.4 & 19 & - & - \\
\hline $\begin{array}{l}\text { Lake Delavan, } \\
\text { Wisconsin }\end{array}$ & 3.4 & 12 & 16 & 37 \\
\hline $\begin{array}{l}\text { Illinois River, } \\
\text { Starved Rock State Park }\end{array}$ & 4.1 & 8 & - & - \\
\hline $\begin{array}{l}\text { Lake Shafer, } \\
\text { Indiana }\end{array}$ & 2.9 & 23 & 5.9 & 28 \\
\hline $\begin{array}{l}\text { Lake Michigan, } \\
\text { St. Joseph, Michigan }\end{array}$ & 3.6 & 14 & - & - \\
\hline $\begin{array}{l}\text { Pipestone Lake, } \\
\text { Michigan }\end{array}$ & 2.2 & 25 & 1.5 & 11 \\
\hline Average & 3.3 & 17 & 8 & 25 \\
\hline
\end{tabular}


NONVOLATILE RADIOACTIVITIES IN SURFACE SOIL ON ANL SITE, 1957

\begin{tabular}{|l|c|c|c|r|r|}
\hline & & \multicolumn{2}{c|}{$\begin{array}{c}\text { Alpha } \\
\text { Activity, } \\
\text { Monthected }\end{array}$} & $\begin{array}{c}\text { No. of } \\
\text { Samples }\end{array}$ & \multicolumn{2}{c|}{$\begin{array}{c}\text { Beta } \\
\mu \mu \text { ctivity, } \\
\end{array}$} & & Max. & Av. & Max. & Av. \\
\cline { 3 - 6 } & & 27 & 23 & 62 & 57 \\
\hline January & 4 & 31 & 23 & 76 & 62 \\
March & 20 & 30 & 24 & 67 & 58 \\
May & 4 & 33 & 25 & 95 & 70 \\
June & 7 & 29 & 26 & 229 & 101 \\
August & 7 & 21 & 17 & 85 & 67 \\
October & 2 & 39 & 26 & 106 & 76 \\
December & 3 & 39 & 24 & 229 & 70 \\
\hline Annual & & & & & \\
Summary & 47 & 39 & \\
\hline
\end{tabular}

about $20 \mu \mu \mathrm{c} / \mathrm{g}$ higher than soil obtained 50 yards from the shed. The alpha and beta activities in this sample did not decay. This increase was due to uranium contamination from the shed and has been observed in previous years.

The samples collected in March were obtained from the immediate vicinity of the $E B W R$ building as part of a study of the activity leaving the reactor. Samples were collected 10,50,100,250, and 500 yards from the building in four directions. The alpha and beta activities in all samples were normal, ranging from $18-31 \mu \mu \mathrm{c} \alpha / \mathrm{g}$ and 54-76 $\mu \mu \mathrm{c} \beta / \mathrm{g}$; no trend with direction or distance was noted. The results will be used as a basis for comparison with similar surveys in the future to determine if the reactor is affecting the radioactivity of its environment.

Soil from locations within 25 miles of the Laboratory (Table XXIV) contained an average of 23 and 70 micromicrocuries of alpha and beta activities, respectively. These values were essentially the same as those found on the Laboratory site. The alpha activities were similar to those found in previous years from the same locations, although the beta values averaged about $15 \mu \mu \mathrm{c} / \mathrm{g}$ higher than in the past three years. The latter increase is attributed to increased fission product activity from fallout in some of the samples. The beta activities in such samples decayed to normal values in about four months. The total activities in soil from the reference sites (Table XXV) were normal and have not changed significantly during the past few years. No appreciable amount of fallout was detected in any of the samples during 1957. 
TABLE XXIV

NONVOLATILE RADIOACTIVITY IN SURFACE SOIL NEAR ANL, 1957

\begin{tabular}{|c|c|c|c|c|c|c|}
\hline \multirow[t]{2}{*}{$\begin{array}{c}\text { Distance From } \\
\text { ANL (miles) }\end{array}$} & \multirow[t]{2}{*}{$\begin{array}{c}\text { Month } \\
\text { Collected }\end{array}$} & \multirow[t]{2}{*}{$\begin{array}{l}\text { No. of } \\
\text { Samples }\end{array}$} & \multicolumn{2}{|c|}{$\begin{array}{c}\text { Alpha } \\
\text { Activity } \\
\mu \mu \mathrm{c} / \mathrm{gram}\end{array}$} & \multicolumn{2}{|c|}{$\begin{array}{c}\text { Beta } \\
\text { Activity } \\
\mu \mu \mathrm{c} / \mathrm{gram}\end{array}$} \\
\hline & & & Max. & Av. & Max. & Av. \\
\hline 10 & April & 5 & 21 & 18 & 67 & 58 \\
\hline 10 & June & 6 & 30 & 23 & 139 & 72 \\
\hline 10 & October & 7 & 26 & 20 & 310 & 95 \\
\hline 10 & December & 3 & 21 & 18 & 70 & 60 \\
\hline 10 (average) & & & \multicolumn{2}{|c|}{20} & \multicolumn{2}{|c|}{75} \\
\hline 25 & April & 5 & 29 & 21 & 65 & 59 \\
\hline 25 & June & 6 & 26 & 19 & 77 & 55 \\
\hline 25 & October & 7 & 23 & 21 & 97 & 74 \\
\hline 25 & December & 3 & 36 & 24 & 111 & 76 \\
\hline 25 (average) & & & \multicolumn{2}{|c|}{21} & \multicolumn{2}{|c|}{65} \\
\hline $\begin{array}{l}\text { Annual } \\
\text { Average }\end{array}$ & & & \multicolumn{2}{|c|}{23} & \multicolumn{2}{|c|}{70} \\
\hline
\end{tabular}

TABLE XXV

NONVOLATILE RADIOACTIVITIES IN SURFACE SOIL AT REFERENCE SITES, 1957

\begin{tabular}{|l|c|c|c|c|}
\hline & \multicolumn{2}{|c|}{ June } & \multicolumn{2}{c|}{ December } \\
\cline { 2 - 5 } & $\mu \mu \mathrm{c} \alpha / \mathrm{g}$ & $\mu \mu \mathrm{c} \beta / \mathrm{g}$ & $\mu \mu \mathrm{c} \alpha / \mathrm{g}$ & $\mu \mu \mathrm{c} \beta / \mathrm{g}$ \\
\hline Geneva, Wisconsin & 19 & 46 & - & - \\
Delavan, Wisconsin & 17 & 45 & 15 & 54 \\
Starved Rock, Illinois & 22 & 37 & 19 & 45 \\
Lake Shafer, Indiana & 14 & 46 & 16 & 44 \\
St. Joseph, Michigan & 11 & 36 & 13 & 52 \\
Pipestone Lake, Michigan & 6 & 31 & 6 & 33 \\
\hline Average & 15 & 40 & 14 & 46 \\
\hline
\end{tabular}




\section{F. Plants}

Plant collection was limited to grass, since it was available at all locations. The results of analysis of total activity of grass during 1957 are summarized in Tables XXVI, XXVII, and XXVIII. Concentrations are given in terms of the oven-dried samples. The alpha activity averaged approximately $1.6 \mu \mu \mathrm{c} / \mathrm{g}$ at all locations, and the individual values were in the range normally found. The average beta activities on and near the ANL site were 170 to $132 \mu \mu \mathrm{c} / \mathrm{g}$, respectively, or about twice that found in previous years. This increase in the average resulted from the unusually high results in March, August, and October for the on-site samples and in April and October for the off-site samples. For the August and October samples, the increases in beta activity corresponded to increases in air and rain activity: the se were evidently due to fallout. The high results for samples collected on the site late in March and near the Laboratory early in April are difficult to explain. The beta activity in air samples increased from about $1.2 \mu \mu \mathrm{c} / \mathrm{M}^{3}$ during the first $2 \frac{1}{2}$ months of the year to about $2 \mu \mu \mathrm{c} / 1$ during the two weeks prior to the March sample collection. In addition, a heavy rainfall ( 0.7 in.) on March 19 contained approximately $1500 \mu \mu \mathrm{c} / 1$ of beta activity about 30 days old. The fission products in this rain may have been concentrated by the gras to an unusual extent, although in the past this amount of rain-out has not produced beta activity increases of the magnitude found in March and April of this year.

TABLE XXVI

NONVOLATILE RADIOACTIVITIES IN GRASS ON ANL SITE, 1957

\begin{tabular}{|c|c|c|c|c|c|}
\hline \multirow[t]{2}{*}{$\begin{array}{c}\text { Date } \\
\text { Collected }\end{array}$} & \multirow[t]{2}{*}{$\begin{array}{c}\text { No. of } \\
\text { Samples }\end{array}$} & \multicolumn{2}{|c|}{$\begin{array}{c}\text { Alpha } \\
\text { Activity, } \\
\mu \mu c / g\end{array}$} & \multicolumn{2}{|c|}{$\begin{array}{c}\text { Alpha } \\
\text { Activity } \\
\mu \mu \mathrm{c} / \mathrm{g}\end{array}$} \\
\hline & & $\operatorname{Max}$ & Av. & Max. & Av. \\
\hline January 28,29 & 4 & 0.9 & 0.8 & 106 & 86 \\
\hline March 28, 29 & 11 & 7.4 & 2.9 & 293 & 199 \\
\hline June 28 & 7 & 1.1 & 0.8 & 128 & 96 \\
\hline August 7 & 7 & 1.1 & 0.8 & 394 & 283 \\
\hline October 2 & 2 & 2.7 & 1.5 & 191 & 178 \\
\hline December 26 & 6 & 3.6 & 1.7 & 76 & 62 \\
\hline Average & & \multicolumn{2}{|c|}{1.6} & \multicolumn{2}{|c|}{170} \\
\hline
\end{tabular}


TABLE XXVII

NONVOLATILE RADIOACTIVITIES IN GRASS

NEAR ANL, 1957

\begin{tabular}{|c|c|c|c|c|c|c|}
\hline \multirow[t]{2}{*}{$\begin{array}{c}\text { Distance From } \\
\text { ANL (miles) }\end{array}$} & \multirow[t]{2}{*}{ Date } & \multirow[t]{2}{*}{$\begin{array}{l}\text { No. of } \\
\text { Samples }\end{array}$} & \multicolumn{2}{|c|}{$\begin{array}{l}\text { Alpha } \\
\text { Activity, } \\
\mu \mu \mathrm{c} / \mathrm{g}\end{array}$} & \multicolumn{2}{|c|}{$\begin{array}{c}\text { Beta } \\
\text { Activity, } \\
\mu \mu \mathrm{c} / \mathrm{g}\end{array}$} \\
\hline & & & Max. & $\mathrm{Av}$. & Max. & Av. \\
\hline 10 & April & 6 & 5.0 & 2.6 & 277 & 182 \\
\hline 10 & June & 6 & 2.1 & 1.0 & 81 & 73 \\
\hline 10 & October & 6 & 1.7 & 0.9 & 235 & 178 \\
\hline 10 & December & 3 & 2.0 & 1.4 & 66 & 58 \\
\hline 10 (average) & & & \multicolumn{2}{|c|}{1.5} & \multicolumn{2}{|c|}{132} \\
\hline 25 & April & 4 & 4.3 & 3.6 & 254 & 198 \\
\hline 25 & June & 5 & 0.8 & 0.6 & 113 & 80 \\
\hline 25 & October & 4 & 1.6 & 1.0 & 210 & 183 \\
\hline 25 & December & 3 & 2.7 & 1.6 & 71 & 58 \\
\hline 25 (average) & & & \multicolumn{2}{|c|}{1.6} & \multicolumn{2}{|c|}{131} \\
\hline $\begin{array}{l}\text { Annual } \\
\text { Average }\end{array}$ & & & \multicolumn{2}{|c|}{1.6} & \multicolumn{2}{|c|}{132} \\
\hline
\end{tabular}

TABLE XXVIII

NONVOLATILE RADIOACTIVITIES IN GRASS

FROM REFERENCE SITES, 1957

\begin{tabular}{|l|c|c|c|c|}
\hline \multirow{2}{*}{ Location } & \multicolumn{2}{c|}{ June } & \multicolumn{2}{c|}{ December } \\
\cline { 2 - 5 } & $\mu \mu \mathrm{c} \alpha / \mathrm{g}$ & $\mu \mu \mathrm{c} \beta / \mathrm{g}$ & $\mu \mu \mathrm{c} \alpha / \mathrm{g}$ & $\mu \mu \mathrm{c} \beta / \mathrm{g}$ \\
\hline Geneva, Wisconsin & 1.6 & 56 & 0.8 & 43 \\
Delavan, Wisconsin & 1.7 & 72 & - & - \\
Starved Rock, Illinois & 1.9 & 78 & 4.2 & 88 \\
Shafer Lake, & 0.7 & 48 & - & - \\
Monticello, Indiana & 1.9 & 78 & 0.5 & 26 \\
St. Joseph, Michigan & 0.2 & 40 & - & - \\
Pipestone Lake, Michigan & 1.3 & 62 & 1.8 & 52 \\
\hline Average & & & & \\
\hline
\end{tabular}


The beta activity in all of the samples, including those collected in March and April, decayed to normal values $(40-80 \mu \mu \mathrm{c} / \mathrm{g})$, regardless of the initial activity, with the most active samples decaying at the highest rates.

\section{G. Animals}

Only a limited number of animals were collected and analyzed. The value of the results obtainable from animal samples was not believed to justify an extensive sampling program similar to that undertaken for the other types of samples.

Two fish samples were collected from the ponds on the ANL site in September. A group of several bullheads contained $0.1 \mu \mu \mathrm{c} \alpha / \mathrm{g}$ and $9.6 \mu \mu \mathrm{c} \beta / \mathrm{g}$ and a sample of bluegills contained $0.1 \mu \mu \mathrm{c} \alpha / \mathrm{g}$ and $12.3 \mu \mu \mathrm{c} \beta / \mathrm{g}$. These results were similar to the total activities found in fish from the same ponds in previous years. Earlier results ranged from 0.2 to $1.7 \mu \mu \mathrm{c} \alpha / \mathrm{g}$ and 16 to $23 \mu \mu \mathrm{c} \beta / \mathrm{g}$.

An aged deer, from a herd that lives on the ANL site, was obtained in May and several organs were analyzed for total activity. Samples of leg bone, leg muscle, lung, liver, kidney, and spleen were counted. The alpha activities ranged from $0.2 \mu \mu \mathrm{c} / \mathrm{g}$ in the spleen to $2.1 \mu \mu \mathrm{c} / \mathrm{g}$ in the bone: the beta activities ranged from $2.0 \mu \mu \mathrm{c} / \mathrm{g}$ in the kidney to $8.3 \mu \mu \mathrm{c} / \mathrm{g}$ in the spleen. The results were considered to be normal. All animal results are given in terms of the air-dried samples. 